ESAIM: COCV 22 (2016) 1054-1077

DOI: $10.1051 / \mathrm{cocv} / 2016045$
ESAIM: Control, Optimisation and Calculus of Variations

www.esaim-cocv.org

\title{
ON THE CONTROLLABILITY OF DIFFUSION PROCESSES ON A SPHERE: A NUMERICAL STUDY
}

\author{
D. Assaely León Velasco ${ }^{1}$, Roland Glowinski ${ }^{2,3}$ \\ AND L. HÉCtor JuÁrez VAlencia ${ }^{1}$
}

\begin{abstract}
The main goal of this article is to study computationally the controllability of a diffusion process on the surface of a sphere in $\mathbb{R}^{3}$. To achieve this goal, we employ a methodology combining finite differences for the time discretization, finite elements for the space approximation, and a conjugate gradient algorithm for the iterative solution of the discrete control problems. The results of numerical experiments, obtained using the above methodology, will be presented. Furthermore, the null-controllability properties of the diffusion model under consideration will be also studied computationally.
\end{abstract}

Mathematics Subject Classification. 49K20, 58E25, 65K10, 65M60,93M05, 93C20.

Received June 6, 2016. Accepted June 7, 2016.

\section{INTRODUCTION}

Many physico-chemical phenomena modeled by partial differential equations take place on the surface of planet Earth; it makes sense therefore to attempt controlling some of them (pollution for example). This evidence leads naturally to control and controllability problems for surfaces of $\mathbb{R}^{3}$, spheres in particular. Looking at the literature shows that, for example, the control of diffusion processes on surfaces of $\mathbb{R}^{3}$ has not attracted much attention, yet, despite the fact that such problems have potentially many applications, notable exceptions being, among few others, $[4,11,14,15]$. Actually, the origin of this article is the null-controllability results for the heat equation proved in [11], not only for bounded domains of $\mathbb{R}^{d}$, but also for Riemannian manifolds, the usual Laplace operator being replaced then by the Beltrami Laplacian. One of our goals in this article is to compute the control of minimal $\mathcal{L}^{2}$-norm realizing the null-controllability at a given time $T$. This problem being more complicated than what it looks like (as shown in [16] for example), we decided to approximate it via a sequence of penalized problems, which are relatively easy to solve numerically, by variants of the methods discussed in [3] for the usual heat equation. Albeit computational null-controllability was our driver, we decided to take advantage of the developed methodology to, first, investigate computationally the solution of controllability

Keywords and phrases. Diffusion process, surface of a shere, conjugate gradient, null-controlability, approximate controllability, Laplace-Beltrami operator.

1 Departamento de Matemáticas, Universidad Autónoma Metropolitana Unidad Iztapalapa, Av. San Rafael Atlixco 186, Col. Vicentina, D.F. 09340, Mexico. assaely@xanum.uam.mx; hect@xanum.uam.mx

2 Deparment of Mathematics, University of Houston, 4800 Calhoun, Houston, TX 77004, USA

3 Baptist University, Hong-Kong, P.R. China. roland@math.uh.edu 


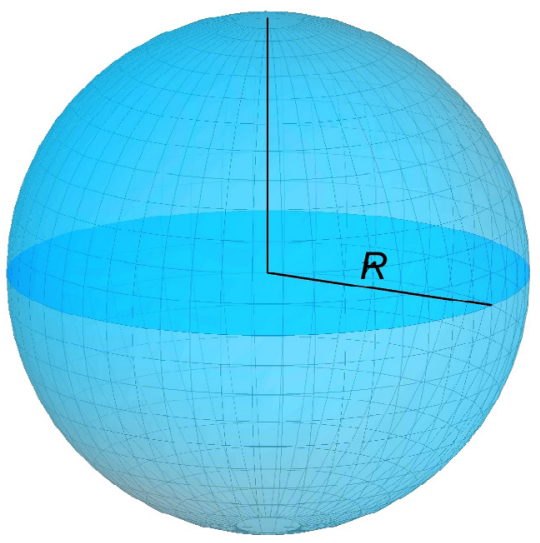

Figure 1. Surface of a sphere.

problems where the target function is different from zero, a much more demanding situation since, in general, diffusion processes are not exactly controllable. In a recent work [12], we considered the same problem but on the surface of a torus, which is easier to parameterize and triangulate than spherical ones. Here we extend the methods discussed in [12] to the solution of controllability problems for diffusion phenomena on spheres in $\mathbb{R}^{3}$.

\section{THE MODEL PROBLEM}

\subsection{Generalities}

As mentioned in Section 1, our goal in this article is to discuss the numerical solution of a controllability problem associated with a diffusion process taking place on the surface of a sphere in $\mathbb{R}^{3}$. We will consider cartesian coordinates to represent this surface in order to avoid the singularities that the usual parameterization (spherical coordinates) introduces. The sphere is visualized in Figure 1 and we denote by $\Sigma$ the surface given by $x^{2}+y^{2}+z^{2}=R^{2}$, where $R$ is the sphere radius.

Let $\omega$ be an open subset of $\Sigma$ (not necessarily connected). The first problem to be considered is an exact controllability one, namely (with $0<T<\infty$ ).

Find $v \in \mathcal{L}^{2}(\omega \times(0, T))$ such that the solution $y$ of the following parabolic initial value problem

$$
\left\{\begin{array}{l}
\int_{\Sigma} \frac{\partial y}{\partial t}(t) z \mathrm{~d} \Sigma+\mu \int_{\Sigma} \nabla_{\Sigma} y(t) \cdot \nabla_{\Sigma} z \mathrm{~d} \Sigma=\int_{\omega} v(t) z \mathrm{~d} \Sigma, \forall z \in \mathcal{H}^{1}(\Sigma), \text { a.e. on }(0, T), \\
y(0)=y_{0},
\end{array}\right.
$$

verifies

$$
y(T)=y_{T},
$$

where:

(i) $\nabla_{\Sigma}$ is the tangential gradient on $\Sigma$;

(ii) $\mathrm{d} \Sigma$ is the infinitesimal surface measure;

(iii) $\mathcal{H}^{1}(\Sigma)=\left\{\left.z\left|z \in \mathcal{L}^{2}(\Sigma), \int_{\Sigma}\right| \nabla_{\Sigma} z\right|^{2} \mathrm{~d} \Sigma<+\infty\right\}$;

(iv) $\mathbf{a} \cdot \mathbf{b}=\sum_{i=1}^{3} a_{i} b_{i}, \forall \mathbf{a}=\left(a_{i}\right)_{i=1}^{3}, \mathbf{b}=\left(b_{i}\right)_{i=1}^{3}$;

(v) $y_{0}$ and $y_{T}$ are given in $\mathcal{L}^{2}(\Sigma)$, and $\mu(>0)$ is a diffusion coefficient;

(vi) $\varphi(t)$ denotes the function $x \longrightarrow \varphi(x, t)$ from $\Sigma$ (or $\omega$ ) into $\mathbb{R}$. 
The above exact controllability problem has no solution, in general. However, we have approximate controllability since the set $\{y(T ; v)\}_{v \in \mathcal{L}^{2}(\omega \times(0, T))}$ is dense in $\mathcal{L}^{2}(\Sigma)$ (see the Chap. 1 of [10] for related results). We will take advantage of the above density results to define, below, an approximately controllable variant of the above exact controllability problem.

Remark 2.1. Suppose that $y_{T}=0$; it follows then from [11] that, $\forall y_{0} \in \mathcal{L}^{2}(\Sigma)$, there exists a control $v \in$ $\mathcal{L}^{2}(\omega \times(0, T))$, such that the solution of the initial value problem $(2.1)$ verifies $y(T)=0$. This property is known as the null-controllability property and holds for sufficiently smooth surfaces of $\mathbb{R}^{d}$ (and bounded Cartesian domains). We will return on the null-controllability property in Section 6.4.

Remark 2.2. The elliptic operator associated with equation (2.1) is clearly $(-1) \mu$ times the Laplace-Beltrami operator (as discussed for example in $[1,2]$ ).

\subsection{Formulation of an approximate controllability problem}

Taking advantage of the density results mentioned in Section 2.1, we approximate the exact controllability problem introduced above by the following one (of the approximation by penalty type):

$$
\left\{\begin{array}{l}
\text { Find } u_{k} \in \mathscr{U}, \text { such that } \\
J_{k}\left(u_{k}\right) \leq J_{k}(v), \forall v \in \mathscr{U},
\end{array}\right.
$$

where $\mathscr{U}=\mathcal{L}^{2}(\omega \times(0, T))$, and the cost functional $J_{k}: \mathscr{U} \rightarrow \mathbb{R}$ is defined by

$$
J_{k}(v)=\frac{1}{2} \int_{\omega \times(0, T)}|v|^{2} \mathrm{~d} \Sigma \mathrm{d} t+\frac{k}{2} \int_{\Sigma}\left|y(T)-y_{T}\right|^{2} \mathrm{~d} \Sigma,
$$

with $k$ a fixed positive number, and the function $y$ obtained from the control $v$ via the solution of the initial value problem (2.1). It follows from, e.g., [10] that the approximate controllability problem (2.3) has a unique solution, characterized by

$$
D J_{k}\left(u_{k}\right)=0,
$$

where $D J_{k}\left(u_{k}\right)$ is the differential of $J_{k}$ at $u_{k}$. The solution of (2.5) by a conjugate gradient algorithm operating in $\mathscr{U}$ will be discussed in Section 4 , but before we will address the computation of $D J_{k}(v), \forall v \in \mathscr{U}$, a most important issue, indeed.

Remark 2.3. Using results from [10], one can show that if one denotes by $y_{k}$ the solution of (2.1) associated with $u_{k}$, then

$$
\lim _{k \rightarrow+\infty} y_{k}(T)=y_{T} \text { in } \mathcal{L}^{2}(\Sigma),
$$

justifying thus taking (2.3) as approximate controllability problem. On the other hand, unless the exact controllability property holds for the target function $y_{T}$ under consideration, we have

$$
\lim _{k \rightarrow+\infty}\left\|u_{k}\right\|_{\mathcal{L}^{2}(\omega \times(0, T))}=+\infty .
$$

A related result is provided by the following: suppose that the exact controllability property holds for the target function $y_{T}$. We have then

$$
\lim _{k \rightarrow+\infty} u_{k}=u \text { in } \mathcal{L}^{2}(\omega \times(0, T)),
$$

where, in (2.6), $u$ is the control of minimal norm in $\mathcal{L}^{2}(\omega \times(0, T))$, realizing the exact controllability. The proof of this last result is included in [12].

Assuming that $y_{T}=0$, the above result justifies using the penalty approach to approximate the control function of minimal norm in $\mathscr{U}$ realizing null-controllability. 


\section{Computation of $D J_{k}(v)$. Optimality Conditions}

Let $v \in \mathscr{U}$, proceeding as in [12], we can easily show that

$$
D J_{k}(v)=v+\left.p\right|_{\omega \times(0, T)},
$$

where $p$ is the solution of the backward (in time) initial value problem

$$
\left\{\begin{array}{l}
-\int_{\Sigma} \frac{\partial p}{\partial t}(t) z \mathrm{~d} \Sigma+\mu \int_{\Sigma} \nabla_{\Sigma} p(t) \cdot \nabla_{\Sigma} z \mathrm{~d} \Sigma=0, \forall z \in \mathcal{H}^{1}(\Sigma), \text { a.e. on }(0, T), \\
p(T)=k\left(y(T)-y_{T}\right) .
\end{array}\right.
$$

This equation is the adjoint (or co-state) equation of the state equation:

$$
\left\{\begin{array}{l}
\int_{\Sigma} \frac{\partial y}{\partial t}(t) z \mathrm{~d} \Sigma+\mu \int_{\Sigma} \nabla_{\Sigma} y(t) \cdot \nabla_{\Sigma} z \mathrm{~d} \Sigma=\int_{\omega} v(t) z \mathrm{~d} \Sigma, \forall z \in \mathcal{H}^{1}(\Sigma), \text { a.e. on }(0, T), \\
y(0)=y_{0},
\end{array}\right.
$$

Of course, this last equation must be solved first in order to compute $y(T)$ in equation (3.2).

Let $u_{k}$ be the unique solution of the control problem (2.3), and denote by $y_{k}$ and $p_{k}$ the associated solution of (2.1) and (3.2), respectively. It follows from the previous discussion that $u_{k}$ is characterized by the following relations (the optimality system):

$$
u_{k}+\left.p_{k}\right|_{\omega \times(0, T)}=0
$$

with

$$
\left\{\begin{array}{l}
\int_{\Sigma} \frac{\partial y_{k}}{\partial t}(t) z \mathrm{~d} \Sigma+\mu \int_{\Sigma} \nabla_{\Sigma} y_{k}(t) \cdot \nabla_{\Sigma} z \mathrm{~d} \Sigma=\int_{\omega} u_{k}(t) z \mathrm{~d} \Sigma, \forall z \in \mathcal{H}^{1}(\Sigma), \text { a.e. on }(0, T), \\
y_{k}(0)=y_{0},
\end{array}\right.
$$

and

$$
\left\{\begin{array}{l}
-\int_{\Sigma} \frac{\partial p_{k}}{\partial t}(t) z \mathrm{~d} \Sigma+\mu \int_{\Sigma} \nabla_{\Sigma} p_{k}(t) \cdot \nabla_{\Sigma} z \mathrm{~d} \Sigma=0, \forall z \in \mathcal{H}^{1}(\Sigma), \text { a.e. on }(0, T), \\
p_{k}(T)=k\left(y_{k}(T)-y_{T}\right) .
\end{array}\right.
$$

Relations (3.4)-(3.6) clearly suggest the following approach (of the fixed point type) to solve the control problem (2.3):

(i) Let $u^{0}$ be a guess of $u_{k}$.

(ii) Denote by $y^{0}$ the solution of $(2.1)$ associated with $u^{0}$.

(iii) Denote by $p^{0}$ the solution of $(3.2)$ associated with $y^{0}$.

(iv) If $u^{0}+\left.p^{0}\right|_{\omega \times(0, T)}$ is small enough in $\mathscr{U}$ take $u_{k}=-\left.p^{0}\right|_{\omega \times(0, T)}$; otherwise use appropriately the residual $u^{0}+\left.p^{0}\right|_{\omega \times(0, T)}$ to correct $u^{0}$, and repeat the process.

In Section 4 we will show that the above program can be achieved using a conjugate gradient algorithm operating in the space $\mathscr{U}$.

Remark 3.1. An alternative to the above approach (a dual of it in some sense, as shown in the Chapter 1 of [10]) can be defined as follows:

(i) Let $e^{0}$ be a guess of $p_{k}(T)$.

(ii) Denote by $p^{0}$ the solution of (3.2) verifying $p^{0}(T)=e^{0}$.

(iii) Denote by $y^{0}$ the solution of (2.1) associated with $u^{0}=-\left.p^{0}\right|_{\omega \times(0, T)}$. 
(iv) If $e^{0}-k\left(y^{0}(T)-y_{T}\right)$ is small enough in $\mathcal{L}^{2}(\Sigma)$ take $u_{k}=u^{0}$; otherwise use appropriately the residual $e^{0}-k\left(y^{0}(T)-y_{T}\right)$ to correct $e^{0}$, and repeat the process.

This alternative approach can be implemented via a conjugate gradient algorithm operating in $\mathcal{L}^{2}(\Sigma)$. Variants of it have been tested in $[3,10]$ to solve numerically approximate controllability problems for the heat equation in bounded domains of $\mathbb{R}^{2}$. In this article, we will solve directly problem (2.3) (the primal problem) using a conjugate gradient algorithm operating in the control space $\mathscr{U}$. The main reasons for preferring the direct approach to the dual one are that (a) it is conceptually simpler, and (b) it can be easily extended to nonlinear diffusion models, unlike the dual approach.

\section{Conjugate Gradient solution of the minimization Problem (2.3)}

Problem (2.3) is a well-posed minimization problem in the control space $\mathscr{U}\left(=\mathcal{L}^{2}(\omega \times(0, T))\right.$, a real Hilbert space for the inner-product $\{v, w\} \rightarrow \int_{\omega \times(0, T)} v w \mathrm{~d} \Sigma \mathrm{d} t$. It is therefore a particular case of those minimization problems in Hilbert spaces whose conjugate gradient solution is discussed in, e.g., the Chapter 1 of [10] and the Chapter 3 of [8] (see also the many references therein). Taking into account the results of Section 3, relation (3.1) in particular, the solution of problem (2.3) can be achieved by the following conjugate gradient algorithm (for notation convenience we denote by $Y_{0}$ the initial value we denoted by $y_{0}$ in the previous sections):

\section{Step 1. Initialization: Given}

$$
u_{0} \text { in } \mathscr{U} \text {, }
$$

solve the following two problems:

$$
\begin{aligned}
& \left\{\begin{array}{l}
\int_{\Sigma} \frac{\partial y_{0}}{\partial t}(t) z \mathrm{~d} \Sigma+\mu \int_{\Sigma} \nabla_{\Sigma} y_{0}(t) \cdot \nabla_{\Sigma} z \mathrm{~d} \Sigma=\int_{\omega} u_{0}(t) z \mathrm{~d} \Sigma, \forall z \in \mathcal{H}^{1}(\Sigma), \text { a.e. on }(0, T), \\
y_{0}(0)=Y_{0},
\end{array}\right. \\
& \left\{\begin{array}{l}
-\int_{\Sigma} \frac{\partial p_{0}}{\partial t}(t) z \mathrm{~d} \Sigma+\mu \int_{\Sigma} \nabla_{\Sigma} p_{0}(t) \cdot \nabla_{\Sigma} z \mathrm{~d} \Sigma=0, \forall z \in \mathcal{H}^{1}(\Sigma), \text { a.e. on }(0, T), \\
p_{0}(T)=k\left(y_{0}(T)-y_{T}\right) .
\end{array}\right.
\end{aligned}
$$

and set $g_{0}=u_{0}+\left.p_{0}\right|_{\omega \times(0, T)}$.

If $\int_{\omega \times(0, T)}\left|g_{0}\right|^{2} \mathrm{~d} \Sigma \mathrm{d} t / \max \left\{1, \int_{\omega \times(0, T)}\left|u_{0}\right|^{2} \mathrm{~d} \Sigma \mathrm{d} t\right\} \leq t o l$, take $u_{k}=u_{0}$ and stop; otherwise set

$$
d_{0}=g_{0}
$$

Step 2. Descent: Then for $q \geq 0$, assuming that $u_{q}, g_{q}$, and $d_{q}$ are known, the last two different from 0 , we compute $u_{q+1}, g_{q+1}$, and if necessary, $d_{q+1}$ as follows:

Solve

$$
\begin{aligned}
& \left\{\begin{array}{l}
\int_{\Sigma} \frac{\partial \bar{y}_{q}}{\partial t}(t) z \mathrm{~d} \Sigma+\mu \int_{\Sigma} \nabla_{\Sigma} \bar{y}_{q}(t) \cdot \nabla_{\Sigma} z \mathrm{~d} \Sigma=\int_{\omega} d_{q}(t) z \mathrm{~d} \Sigma, \forall z \in \mathcal{H}^{1}(\Sigma), \text { a.e. on }(0, T), \\
\bar{y}_{q}(0)=0,
\end{array}\right. \\
& \left\{\begin{array}{l}
-\int_{\Sigma} \frac{\partial \bar{p}_{q}}{\partial t}(t) z \mathrm{~d} \Sigma+\mu \int_{\Sigma} \nabla_{\Sigma} \bar{p}_{q}(t) \cdot \nabla_{\Sigma} z \mathrm{~d} \Sigma=0, \forall z \in \mathcal{H}^{1}(\Sigma), \text { a.e. on }(0, T), \\
\bar{p}_{q}(T)=k \bar{y}_{q}(T),
\end{array}\right.
\end{aligned}
$$

and set

$$
\bar{g}_{q}=d_{q}+\left.\bar{p}_{q}\right|_{\omega \times(0, T)}
$$


Compute $\alpha_{q}=\int_{\omega \times(0, T)}\left|g_{q}\right|^{2} \mathrm{~d} \Sigma \mathrm{d} t / \int_{\omega \times(0, T)} d_{q} \bar{g}_{q} \mathrm{~d} \Sigma \mathrm{d} t$, and then

$$
\begin{aligned}
& u_{q+1}=u_{q}-\alpha_{q} d_{q}, \\
& g_{q+1}=g_{q}-\alpha_{q} \bar{g}_{q} .
\end{aligned}
$$

Step 3. Testing the convergence. Construction of the new descent direction:

If $\int_{\omega \times(0, T)}\left|g_{q+1}\right|^{2} \mathrm{~d} \Sigma \mathrm{d} t / \max \left\{\int_{\omega \times(0, T)}\left|g_{0}\right|^{2} \mathrm{~d} \Sigma \mathrm{d} t, \int_{\omega \times(0, T)}\left|u_{q+1}\right|^{2} \mathrm{~d} \Sigma \mathrm{d} t\right\} \leq t o l$ take $u_{k}=u_{q+1}$; otherwise, compute

$$
\beta_{q}=\int_{\omega \times(0, T)}\left|g_{q+1}\right|^{2} \mathrm{~d} \Sigma \mathrm{d} t / \int_{\omega \times(0, T)}\left|g_{q}\right|^{2} \mathrm{~d} \Sigma \mathrm{d} t
$$

and

$$
d_{q+1}=g_{q+1}+\beta_{q} d_{q}
$$

Do $q+1 \longrightarrow q$ and return to Step 2 .

Concerning the choice of $t o l$, following the Chapter 3 of [8], we advocate to take $t o l=10^{-d}$ where $d$ is the number of digits used for the floating point representation of the real numbers in the computer platform we use for our computations.

By a slight variant of the analysis done in the Chapter 1 of [10] for the "ordinary heat equation", one can prove that, for a given value of $t o l$, the number of iterations, necessary to achieve the convergence of the above algorithm, varies like $\sqrt{k}$.

\section{Discretization of the Control Problem. Iterative Solution of the Fully} DISCRETE PROBLEM

\subsection{Time discretization of the control problem (2.3)}

Let $N$ be a positive integer. We define the time discretization step $\Delta t$ as $\Delta t=T / N$. Next, we approximate the control problem (2.3) by

$$
\left\{\begin{array}{l}
\mathbf{u}_{k}^{\Delta t} \in \mathscr{U}^{\Delta t} \\
J_{k}^{\Delta t}\left(\mathbf{u}_{k}^{\Delta t}\right) \leq J_{k}^{\Delta t}(\mathbf{v}), \forall \mathbf{v} \in \mathscr{U}^{\Delta t}
\end{array}\right.
$$

where $\mathscr{U}^{\Delta t}=\left(\mathcal{L}^{2}(\omega)\right)^{N}, \mathbf{u}_{k}^{\Delta t}=\left\{u_{k}^{n}\right\}_{n=1}^{N}, \mathbf{v}=\left\{v^{n}\right\}_{n=1}^{N}$, and the cost functional $J_{k}^{\Delta t}$ is defined by

$$
J_{k}^{\Delta t}(\mathbf{v})=\frac{\Delta t}{2} \sum_{n=1}^{N} \int_{\omega}\left|v^{n}\right|^{2} \mathrm{~d} \Sigma+\frac{k}{2} \int_{\Sigma}\left|y^{N}-y_{T}\right|^{2} \mathrm{~d} \Sigma,
$$

with $\left\{y^{n}\right\}_{n=1}^{N}$ obtained from $\mathbf{v}$ and $y_{0}$ by the solution of:

$$
y^{0}=y_{0},
$$

for $n=1, \ldots, N$

$$
\left\{\begin{array}{l}
y^{n} \in \mathcal{H}^{1}(\Sigma) \\
\int_{\Sigma} \frac{y^{n}-y^{n-1}}{\Delta t} z \mathrm{~d} \Sigma+\mu \int_{\Sigma} \nabla_{\Sigma} y^{n} \cdot \nabla_{\Sigma} z \mathrm{~d} \Sigma=\int_{\omega} v^{n} z \mathrm{~d} \Sigma, \quad \forall z \in \mathcal{H}^{1}(\Sigma),
\end{array}\right.
$$


where $y^{n}$ approximates $y(n \Delta t)$. Problems (5.4) are well-posed elliptic problems; strictly speaking they are not associated with any boundary condition since $\Sigma$ is a surface without boundary.

Using classical convexity arguments, one can easily show that the discrete problem (5.1) has a unique solution, characterized by

$$
D J_{k}^{\Delta t}\left(\mathbf{u}_{k}^{\Delta t}\right)=\mathbf{0}
$$

where $D J_{k}^{\Delta t}$ denotes the differential of $J_{k}^{\Delta t}$. Taking $\{\mathbf{v}, \mathbf{w}\} \rightarrow \Delta t \sum_{n=1}^{N} v^{n} w^{v}\left(=(\mathbf{v}, \mathbf{w})_{\Delta t}\right)$ as inner-product over $\mathscr{U}^{\Delta t}$, we can show that

$$
\left(D J_{k}^{\Delta t}(\mathbf{v}), \mathbf{w}\right)_{\Delta t}=\Delta t \sum_{n=1}^{N} \int_{\omega}\left(v^{n}+p^{n}\right) w^{n} \mathrm{~d} \Sigma,
$$

where $\left\{p^{n}\right\}_{n=1}^{N}$ is obtained from $\mathbf{v}$ via the solution of (5.3), (5.4) and of the following backward in time discrete initial value problem (the associated adjoint system):

$$
p^{N+1}=k\left(y^{N}-y_{T}\right),
$$

for $n=N, \ldots, 1$

$$
\left\{\begin{array}{l}
p^{n} \in \mathcal{H}^{1}(\Sigma), \\
\int_{\Sigma} \frac{p^{n}-p^{n+1}}{\Delta t} z \mathrm{~d} \Sigma+\mu \int_{\Sigma} \nabla_{\Sigma} p^{n} \cdot \nabla_{\Sigma} z \mathrm{~d} \Sigma=0, \forall z \in \mathcal{H}^{1}(\Sigma),
\end{array}\right.
$$

where $p^{n}$ approximates $p(n \Delta t)$. There is no basic difficulty at deriving a time-discrete analogue of the conjugate gradient algorithm (4.1)-(4.11), in order to solve the discrete control problem (5.1), via the optimality condition (5.5). In order to avoid being repetitious we will not describe this algorithm in this article. Instead, we will describe a fully-discrete analogue of the two above algorithms in Section 5.2, after discussing the space discretization of problem (5.1).

Remark 5.1. To time-discretize the parabolic problems (2.1) and (3.2), we have used the backward Euler scheme, obtaining thus (5.3), (5.4) and (5.7), (5.8). This implicit scheme is only first order accurate, but is stiff A-stable, robust and preserves the maximum principle if combined with appropriate space approximations. In [3] a second order accurate two step backward implicit scheme has been used to solve related controllability problems for the classical heat equation; this scheme could have been used here also. The main reasons we did not do it are: (a) this second order scheme does not preserve the maximum principle, (b) it requires a starting procedure, (c) deriving the associated discrete adjoint system is significantly more complicated than when using the backward Euler's scheme, (d) if $v=0$, as for the continuous model with backward Euler's scheme, the damping of the spectral modes of the solution increases monotonically with the value of the associated eigenvalues of the opposite of the Laplace-Beltrami operator, and (e) (last but not least) a collaboration with NASA engineers working on the real time control of sub-systems of the Space Shuttle and International Space Station has shown us that most often the time-discretization method of choice for these engineers is the forward Euler's scheme with a fixed time-discretization step, chosen small enough to avoid numerical instabilities.

\subsection{Full discretization of the control problem (2.3)}

The time-discretization of problem (2.3) has been addressed in Section 5.1. In order to obtain a spacediscretization, we can approximate $\Sigma$ by a polyhedral surface and proceed as in [1] to approximate the various elliptic problems encountered in (5.4) and (5.8). However, here we shall proceed in a slightly different manner, mainly when computing the surface gradient.

Let $\Sigma_{h}$ be the polyhedral surface that approximates $\Sigma$, as shown in Figure 2. The elements of $\Sigma_{h}$ are assumed to be triangular facets, and we denote by $\mathcal{T}_{h}$ the set they form. Each triangular facet $K \in \mathcal{T}_{h}$, is described by the isoparametric parametrization

$$
x_{h}(\epsilon, \eta)=\sum_{i=1}^{3} x_{i} \varphi_{i}(\epsilon, \eta)
$$




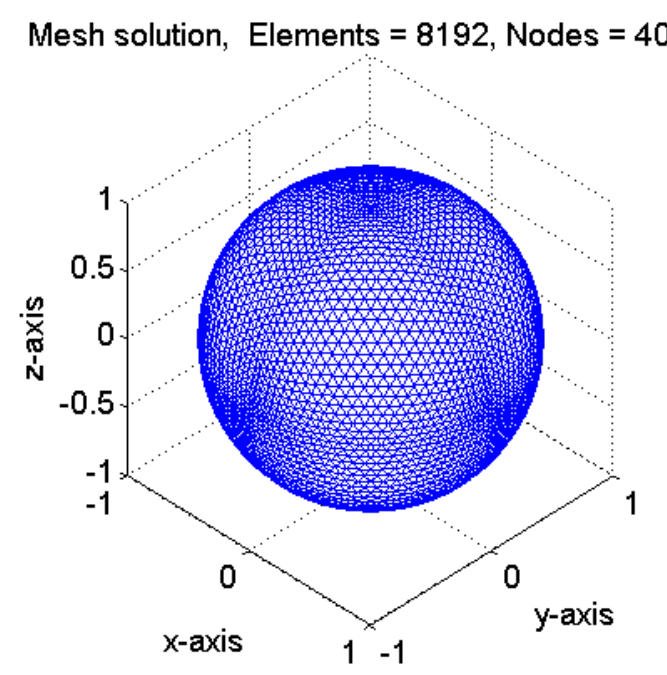

Figure 2. A polyhedral approximation of the sphere of radius 1.

where, $(\epsilon, \eta)$ are the coordinates that define the usual reference element $\widehat{K} \in \mathbb{R}^{2}$, and $x_{i}=\left(x_{1}^{i}, x_{2}^{i}, x_{3}^{i}\right)$, with $i=$ $1,2,3$, are the physical coordinates of the triangular facet $K$. The linear functions $\varphi_{i}(\xi, \eta)$ are the corresponding reference shape functions.

Denote by $h$ the maximum diameter of the elements in $\Sigma_{h}$, we approximate then $H^{1}(\Sigma)$ by

$$
V_{h}=\left\{z\left|z \in H^{1}\left(\Sigma_{h}\right), z\right|_{K} \in \mathbb{P}_{1}, \forall K \in \mathcal{T}_{h}\right\},
$$

where $\mathbb{P}_{1}$ is the space of polynomials of degree $\leq 1$.

Now, we can obtain the discret counterparts of problems (5.4) and (5.8). For instance, the discrete version of (5.4) can be written as follows.

Let $y_{0 h}$ an approximation of $y_{0}$ belonging to $V_{h}$.

For $n=1, \ldots, N$

$$
\left\{\begin{array}{l}
y_{h}^{n} \in V_{h}, \\
\sum_{K \in \mathcal{T}_{h}} \int_{K} \frac{y_{h}^{n}-y_{h}^{n-1}}{\Delta t} z \mathrm{~d} K+\mu \sum_{K \in \mathcal{T}_{h}} \int_{K} \nabla_{K} y_{h}^{n} \cdot \nabla_{K} z \mathrm{~d} K=\sum_{K \in \mathcal{T}_{h}^{\omega}} \int_{K} v_{h}^{n} z \mathrm{~d} K, \quad \forall z \in V_{h} .
\end{array}\right.
$$

In the last integral we are assuming that $\bar{\omega}$ is also the union of triangles of $\mathcal{T}_{h}$, and we denote this set of triangles as $\mathcal{T}_{h}^{\omega}$. The tangential gradient $\nabla_{K} y_{h}^{n}$ can be computed by projecting the usual gradient $\nabla y_{h}^{n}$ on the surface of $K$, that is

$$
\nabla_{K} y_{h}^{n}=\left(\mathbb{I}-\mathbb{P}_{K}\right) \nabla y_{h}^{n}
$$

where $\mathbb{I}$ is the identity matrix in $\mathbb{R}^{3}$ and $\mathbb{P}_{K}=\mathbf{n}_{K} \mathbf{n}_{K}^{t}$ is the orthogonal projection matrix to the normal direction of $K$. This quantity is well defined, since $\nabla y_{h}^{n}$ is a constant vector on every $K \in \mathcal{T}_{h}$ and $\mathbf{n}_{K}$ is the unique unitary vector normal to $K$. Of course, the integral in (5.11) can be computed either in the physical element $K$ or in the reference element $\widehat{K}$ using the parametrization (5.9). Integrals in equation (5.8) are approximated in a similar way. 
To simplify the notation, instead of (5.11) we will write:

For $n=1, \ldots, N$

$$
\left\{\begin{array}{l}
y_{h}^{n} \in \mathcal{H}^{1}\left(\Sigma_{h}\right), \\
\int_{\Sigma_{h}} \frac{y_{h}^{n}-y_{h}^{n-1}}{\Delta t} z \mathrm{~d} \Sigma_{h}+\mu \int_{\Sigma_{h}} \nabla_{\Sigma_{h}} y_{h}^{n} \cdot \nabla_{\Sigma_{h}} z \mathrm{~d} \Sigma_{h}=\int_{\omega_{h}} v_{h}^{n} z \mathrm{~d} \Sigma_{h}, \quad \forall z \in V_{h} .
\end{array}\right.
$$

The previous isoparametric finite element approximation allows us to fully approximate the control problem (2.3) by

$$
\left\{\begin{array}{l}
\mathbf{u}_{k h}^{\Delta t} \in \mathscr{U}_{h}^{\Delta t} \\
J_{k h}^{\Delta t}\left(\mathbf{u}_{k h}^{\Delta t}\right) \leq J_{k h}^{\Delta t}(\mathbf{v}), \forall \mathbf{v} \in \mathscr{U}_{h}^{\Delta t}
\end{array}\right.
$$

where in (5.14) the fully discrete control space $\mathscr{U}_{h}^{\Delta t}$ is defined by

$$
\mathscr{U}_{h}^{\Delta t}=\left\{\mathbf{v}\left|\mathbf{v}=\left\{v_{h}^{n}\right\}_{n=1}^{N}, v_{h}^{n} \in V_{h}\right|_{\bar{\omega}_{h}}\right\},
$$

and is equipped with the inner-product

$$
(\mathbf{v}, \mathbf{w})_{h}^{\Delta t}=\Delta t \sum_{n=1}^{N} \int_{\omega_{h}} v_{h}^{n} w_{h}^{n} \mathrm{~d} \Sigma_{h}
$$

The cost functional $J_{k h}^{\Delta t}$ is now defined by

$$
J_{k h}^{\Delta t}(\mathbf{v})=\frac{\Delta t}{2} \sum_{n=1}^{N} \int_{\omega_{h}}\left|v_{h}^{n}\right|^{2} \mathrm{~d} \Sigma_{h}+\frac{k}{2} \int_{\Sigma_{h}}\left|y_{h}^{N}-y_{T}\right|^{2} \mathrm{~d} \Sigma_{h},
$$

with $\left\{y_{h}^{n}\right\}_{n=1}^{N}$ obtained from $y_{0}$ and $\mathbf{v}$ via the solution of

$$
y_{h}^{0}=y_{0 h}\left(\in V_{h}\right) .
$$

For $n=1, \ldots, N$ solve

$$
\left\{\begin{array}{l}
y_{h}^{n} \in V_{h}, \\
\int_{\Sigma_{h}} \frac{y_{h}^{n}-y_{h}^{n-1}}{\Delta t} z \mathrm{~d} \Sigma_{h}+\mu \int_{\Sigma_{h}} \nabla_{\Sigma_{h}} y_{h}^{n} \cdot \nabla_{\Sigma_{h}} z \mathrm{~d} \Sigma_{h}=\int_{\omega_{h}} v_{h}^{n} z \mathrm{~d} \Sigma_{h}, \quad \forall z \in V_{h} .
\end{array}\right.
$$

Remark 5.2. For the computations whose results will be presented in Section 6, we have employed the trapezoidal rule on each triangle of $\mathcal{T}_{h}$ and $\mathcal{T}_{h}^{\omega}$ to approximate the integrals encountered in (5.16), (5.17) and (5.19), taking advantage of

$$
\int_{\Sigma_{h}}=\sum_{K \in \mathcal{T}_{h}} \int_{K}, \quad \text { and } \quad \int_{\omega_{h}}=\sum_{K \in \mathcal{T}_{h}^{\omega}} \int_{K} .
$$

Proceeding as in Section 3, we can show that the differential $D J_{k h}^{\Delta t}(\mathbf{v})$ of $J_{k h}^{\Delta t}$ at $\mathbf{v}$ is defined by

$$
\left(D J_{h}^{\Delta t}(\mathbf{v}), \mathbf{w}\right)_{h}^{\Delta t}=\Delta t \sum_{n=1}^{N} \int_{\omega_{h}}\left(v_{h}^{n}+p_{h}^{n}\right) w_{h}^{n} \mathrm{~d} \Sigma_{h}, \quad \forall \mathbf{w} \in \mathscr{U}_{h}^{\Delta t},
$$

with $\left\{p_{h}^{n}\right\}_{n=1}^{N}$ obtained from the solution of the fully discrete adjoint problem:

$$
p_{h}^{N+1}=k\left(y_{h}^{N}-y_{T h}\right) .
$$


For $n=N, \ldots, 1$, solve

$$
\left\{\begin{array}{l}
p_{h}^{n} \in V_{h}, \\
\int_{\Sigma_{h}} \frac{p_{h}^{n}-p_{h}^{n+1}}{\Delta t} z \mathrm{~d} \Sigma_{h}+\mu \int_{\Sigma_{h}} \nabla_{\Sigma_{h}} p_{h}^{n} \cdot \nabla_{\Sigma_{h}} z \mathrm{~d} \Sigma_{h}=0, \quad \forall z \in V_{h} .
\end{array}\right.
$$

In (5.21), $y_{T h}$ is an approximation of $y_{T}$ belonging to $V_{h}$. It follows from (5.20) that

$$
D J_{h}^{\Delta t}(\mathbf{v})=\left\{v_{h}^{n}+\left.p_{h}^{n}\right|_{\omega_{h}}\right\}_{n=1}^{N} .
$$

The solution $\mathbf{u}_{k h}^{\Delta t}$ of problem (5.17) is characterized by

$$
D J_{k h}^{\Delta t}\left(\mathbf{u}_{k h}^{\Delta t}\right)=\mathbf{0} .
$$

Remark 5.2 applies also to the integrals in (5.20) and (5.22).

Taking advantage of (5.20) and (5.23), it makes sense to solve the fully discrete control problem (5.14) via the solution of (5.24). This solution can be achieved by a conjugate gradient algorithm, which is nothing but the fully discrete version of algorithm (4.1)-(4.11) discussed in Section 4. This new conjugate gradient algorithm reads as follows:

$$
\text { Given } \mathbf{u}_{0}=\left\{u_{0}^{n}\right\}_{n=1}^{N} \text { belonging to } \mathscr{U}_{h}^{\Delta t}
$$

solve

$$
\left\{\begin{array}{l}
y_{0}^{0}=y_{0 h} \\
\text { for } n=1, \ldots, N, \text { solve } \\
\left\{\begin{array}{l}
y_{0}^{n} \in V_{h}, \\
\int_{\Sigma_{h}} \frac{y_{0}^{n}-y_{0}^{n-1}}{\Delta t} z \mathrm{~d} \Sigma_{h}+\mu \int_{\Sigma_{h}} \nabla_{\Sigma_{h}} y_{0}^{n} \cdot \nabla_{\Sigma_{h}} z \mathrm{~d} \Sigma_{h}=\int_{\omega_{h}} u_{0}^{n} z \mathrm{~d} \Sigma_{h}, \forall z \in V_{h},
\end{array}\right.
\end{array}\right.
$$

and

$$
\left\{\begin{array}{l}
p_{0}^{N+1}=k\left(y_{0}^{N}-y_{T h}\right) \\
\text { for } n=N, \ldots, 1, \text { solve } \\
\left\{\begin{array}{l}
p_{0}^{n} \in V_{h}, \\
\int_{\Sigma_{h}} \frac{p_{0}^{n}-p_{0}^{n+1}}{\Delta t} z \mathrm{~d} \Sigma_{h}+\mu \int_{\Sigma_{h}} \nabla_{\Sigma_{h}} p_{0}^{n} \cdot \nabla_{\Sigma_{h}} z \mathrm{~d} \Sigma_{h}=0, \quad \forall z \in V_{h} .
\end{array}\right.
\end{array}\right.
$$

Define $\mathbf{g}_{0}=\left\{g_{0}^{n}\right\}_{n=1}^{N}$ by

If $\Delta t \sum_{n=1}^{N} \int_{\omega_{h}}\left|g_{0}^{n}\right|^{2} \mathrm{~d} \Sigma_{h} / \max \left\{1, \Delta t \sum_{n=1}^{N} \int_{\omega_{h}}\left|u_{0}^{n}\right|^{2} \mathrm{~d} \Sigma_{h}\right\} \leq t o l$, take $\mathbf{u}_{k h}^{\Delta t}=\mathbf{u}_{0}$; otherwise set

$$
\mathbf{d}_{0}=\mathbf{g}_{0} .
$$

For $q \geq 0$, assuming that $\mathbf{u}_{q}, \mathbf{g}_{q}$, and $\mathbf{d}_{q}$ are known, the last two different from $\mathbf{0}$, we compute $\mathbf{u}_{q+1}, \mathbf{g}_{q+1}$, and, if necessary, $\mathbf{d}_{q+1}$ as follows:

$$
\left\{\begin{array}{l}
\bar{y}_{q}^{0}=0 ; \\
\text { for } n=1, \ldots, N, \text { solve } \\
\left\{\begin{array}{l}
\bar{y}_{q}^{n} \in V_{h}, \\
\int_{\Sigma_{h}} \frac{\bar{y}_{q}^{n}-\bar{y}_{q}^{n-1}}{\Delta t} z \mathrm{~d} \Sigma_{h}+\mu \int_{\Sigma_{h}} \nabla_{\Sigma_{h}} \bar{y}_{q}^{n} \cdot \nabla_{\Sigma_{h}} z \mathrm{~d} \Sigma_{h}=\int_{\omega_{h}} d_{q}^{n} z \mathrm{~d} \Sigma_{h}, \quad \forall z \in V_{h},
\end{array}\right.
\end{array}\right.
$$


and

$$
\left\{\begin{array}{l}
\bar{p}_{q}^{N+1}=k \bar{y}_{q}^{N} ; \\
\text { for } n=N, \ldots, 1, \text { solve } \\
\left\{\begin{array}{l}
\bar{p}_{q}^{n} \in V_{h}, \\
\int_{\Sigma_{h}} \frac{\bar{p}_{q}^{n}-\bar{p}_{q}^{n+1}}{\Delta t} z \mathrm{~d} \Sigma_{h}+\mu \int_{\Sigma_{h}} \nabla_{\Sigma_{h}} \bar{p}_{q}^{n} \cdot \nabla_{\Sigma_{h}} z \mathrm{~d} \Sigma_{h}=0, \quad \forall z \in V_{h} .
\end{array}\right.
\end{array}\right.
$$

Compute

$$
\begin{gathered}
\overline{\mathbf{g}}_{q}=\left\{\bar{g}_{q}^{n}\right\}_{n=1}^{N}=\left\{d_{q}^{n}+\left.\bar{p}_{q}^{n}\right|_{\omega_{h}}\right\}_{n=1}^{N}, \\
\alpha_{q}=\frac{\sum_{n=1}^{N} \int_{\omega_{h}}\left|g_{q}^{n}\right|^{2} \mathrm{~d} \Sigma_{h}}{\sum_{n=1}^{N} \int_{\omega_{h}} \bar{g}_{q}^{n} d_{q}^{n} \mathrm{~d} \Sigma_{h}} \\
\mathbf{u}_{q+1}=\mathbf{u}_{q}-\alpha_{q} \mathbf{d}_{q}, \\
\mathbf{g}_{q+1}=\mathbf{g}_{q}-\alpha_{q} \overline{\mathbf{g}}_{q} . \\
\text { If } \sum_{n=1}^{N} \int_{\omega_{h}}\left|g_{q+1}^{n}\right|^{2} \mathrm{~d} \Sigma_{h} / \max \left\{\sum_{n=1}^{N} \int_{\omega_{h}}\left|g_{0}^{n}\right|^{2} \mathrm{~d} \Sigma_{h}, \sum_{n=1}^{N} \int_{\omega_{h}}\left|u_{q+1}^{n}\right|^{2} \mathrm{~d} \Sigma_{h}\right\} \leq \text { tol, take } \mathbf{u}_{k h}^{\Delta t}=\mathbf{u}_{q+1} ; \text { otherwise } \\
\sum_{n=1}^{N} \int_{\omega_{h}}\left|g_{q+1}^{n}\right|^{2} d \Sigma_{h} \\
\sum_{n=1}^{N} \int_{\omega_{h}}\left|g_{q}^{n}\right|^{2} d \Sigma_{h}
\end{gathered}
$$

and

$$
\mathbf{d}_{q+1}=\mathbf{g}_{q+1}+\beta_{q} \mathbf{d}_{q} .
$$

Do $q+1 \longrightarrow q$ and return to $(5.30)$.

Remark 5.2 still applies for the various integrals encountered in algorithm (5.25)-(5.37). Futher remarks are:

Remark 5.3. The various discrete linear elliptic problems occurring in (5.26), (5.27) and (5.30), (5.31) are all associated with the same matrix, differing only by their right-hand sides. Since the above matrix is symmetric, positive definite and sparse, the associated linear systems can be solved by a sparse Cholesky solver, like the one available in MATLAB ${ }^{\mathrm{R}}$. An alternative to Cholesky is to use a conjugate gradient algorithm initialized by the solution at the previous time step. The backward Euler time discretization scheme that we employ being only first order accurate, using a small $\Delta t$ is recommended, implying that the matrix associated with the backward Euler scheme is not too badly conditioned, authorizing thus the solution of these discrete elliptic problems by a conjugate gradient algorithm preconditioned by the diagonal of the above matrix. In this article we have solved these linear systems using one of those user friendly MATLAB ${ }^{\circledR}$ routines which decide 'by themselves' of the solver which is the most appropriate for the linear system under consideration.

Remark 5.4. The conjugate gradient algorithm (5.25)-(5.37) is a solver for (5.24), a linear problem in $\mathscr{U}_{h}^{\Delta t}$. For large values of the penalization parameter $k$ the condition number of the linear operator associated with $D J_{k h}^{\Delta t}$ is of the order of $k$, implying that the linear problem (5.24) is poorly conditioned making its solution by algorithm (5.25)-(5.37) sensitive to round-off errors. This explains that if we take the stopping criterion tol 
TABLE 1. Summary of convergence results (target function (6.1) and $\omega=\left\{\left(x_{1}, x_{2}, x_{3}\right) \in \Sigma \mid\right.$ $\left.\left.-0.3<x_{3}<0.3\right\}\right)$.

\begin{tabular}{ccccccc}
\hline$h$ & 0.0787 & 0.0201 & 0.0051 & 0.0013 & 0.0201 & 0.0051 \\
$\Delta t$ & $1 / 100$ & $1 / 100$ & $1 / 100$ & $1 / 100$ & $1 / 200$ & $1 / 200$ \\
\hline No. iters & 16 & 20 & 19 & 18 & 20 & 18 \\
Norm $u^{c}$ & 10.3845 & 12.9920 & 14.3885 & 14.5753 & 13.0010 & 14.1877 \\
Rel. error & 0.0531 & 0.0504 & 0.0504 & 0.0522 & 0.0502 & 0.0520 \\
\hline
\end{tabular}

in (5.25)-(5.37) too small, the convergence properties of the above conjugate gradient algorithm will deteriorate. To avoid this situation we have taken tol significantly larger than the value advocated in Section 4 (we took tol of the order of $10^{-5}$ for our computations).

Remark 5.5. The convergence, as $\{h, \Delta t\} \rightarrow\{0,0\}$, of space/time approximations of controllability problems for the heat equations modeling diffusion phenomena in bounded domains of $\mathbb{R}^{d}$ has been addressed in, e.g., $[16,19]$ (see also [10]). Extending the convergence results proved in the three above references to the solutions of the discrete controllability problem (5.14) (and of similar ones associated with other smooth bounded surfaces of $\mathbb{R}^{3}$ ), still has to be done (to the best of our knowledge). We strongly believe that the methods developed in the above references can be (slightly) modified in order to prove the convergence of the solutions of fully discrete controllability problems such as (5.14).

\section{NumERICAL EXAMPLES}

In this section we present the results of numerical experiments, obtained by applying the methodology discussed in the preceding sections to the solution of selected test problems. We consider two types of controllability problems, namely: (i) Approximate controllability problems where the target function $y_{T}$ is different from 0 , and (ii) Null controllability problems (implying thus that $y_{T}=0$ ).

\subsection{Results for a non-zero smooth target function}

The surface $\Sigma$ under consideration is the sphere of radius 1 centered at $(0,0,0)$. The first target function $y_{T}$ to be considered is the smooth function defined by

$$
y_{T}\left(x_{1}, x_{2}, x_{3}\right)=\sin \left(2 x_{1} x_{2}\right)+\cos \left(2 x_{2} x_{3}\right), \quad \forall\left(x_{1}, x_{2}, x_{3}\right) \in \Sigma .
$$

The numerical results reported in Table 1 have been obtained taking $y_{0}=0, \mu=1 / 2$, and $T=2$ in equation (2.1), the domain $\omega$ being the strip defined by $\omega=\left\{\left(x_{1}, x_{2}, x_{3}\right) \in \Sigma \mid-0.3<x_{3}<0.3\right\}$. Several values of the discretization parameters $h$ and $\Delta t$ have been employed to approximate the control problem (2.3), $h$ being the length of the largest edge $(\mathrm{s})$ of the triangulation $\mathcal{T}_{h}$. We took $t o l=10^{-5}$ for the stopping criterion of the conjugate gradient algorithm (5.25)-(5.37), using $\mathbf{u}_{0}=\mathbf{0}$ as initial guess and $k=10^{8}$ as penalty parameter. Related numerical results have been reported in Table 1, where $u^{c}$ (resp., $y^{c}$ ) denotes the computed optimal control (resp., the associated solution of (5.3)-(5.4)), No. iters denotes the number of conjugate gradient iterations necessary to achieve convergence, $N$ orm $u^{c}=\left\|u^{c}\right\|_{\mathcal{L}^{2}(\omega \times(0, T))}$ and Rel. error $=\left\|y_{T}-y^{c}(T)\right\|_{\mathcal{L}^{2}(\Sigma)} /\left.\left\|y_{T}\right\|\right|_{\mathcal{L}^{2}(\Sigma)}$.

Table 1 shows that for $h$ small enough the solution does not vary much with $\Delta t$, suggesting convergence with respect to space-time discretization. In particular, in all cases we investigated, $y^{c}(T)$ differs from $y_{T}$ by $5 \%$ approximately.

We used $h=0.0051$ and $\Delta t=1 / 100$, to investigate the influence of the penalty parameter $k$ on the convergence, for four sub-domains $\omega$ (including the above one), the other parameters and data being as above (here $|\omega|$ and $|\Sigma|$ denotes, respectively, the measures of $\omega$ and $\Sigma$; we have thus $|\Sigma|=4 \pi$ ). The results have been reported on Table 2 and show, among other things, that $\|\left. u^{c}\right|_{\mathcal{L}^{2}(\omega \times(0, T))}$ increases with $1 /|\omega|$. Actually, 
TABle 2. Numerical results for different sub-domains $\omega$ (target function (6.1)).

\begin{tabular}{|c|c|c|c|c|c|}
\hline$\omega$ & $|\omega| /|\Sigma|$ & $k$ & No. Iters & Norm $u^{c}$ & Rel. error \\
\hline \multirow{4}{*}{$\Sigma$} & \multirow{4}{*}{1} & $10^{4}$ & 4 & 6.8156 & 0.0010 \\
\hline & & $10^{6}$ & 4 & 6.8253 & $7.5202 \times 10^{-4}$ \\
\hline & & $10^{8}$ & 4 & 5.0279 & $7.3486 \times 10^{-4}$ \\
\hline & & $10^{10}$ & 4 & 5.0279 & $7.3486 \times 10^{-4}$ \\
\hline \multirow{3}{*}{$\left(x_{1}, x_{2}, x_{3}\right) \in \Sigma \mid x_{3}>0$} & \multirow{3}{*}{0.5} & $10^{4}$ & 28 & 16.5423 & 0.0684 \\
\hline & & $10^{6}$ & 34 & 19.3445 & 0.0567 \\
\hline & & $10^{8}$ & 34 & 19.3586 & 0.0566 \\
\hline \multirow{3}{*}{$\left(x_{1}, x_{2}, x_{3}\right) \in \Sigma \mid-0.3<x_{3}<0.3$} & \multirow{3}{*}{0.3} & $10^{4}$ & 17 & 13.5167 & 0.0537 \\
\hline & & $10^{6}$ & 19 & 14.3812 & 0.0505 \\
\hline & & $10^{8}$ & 19 & 14.3885 & 0.0504 \\
\hline \multirow{3}{*}{$\left(x_{1}, x_{2}, x_{3}\right) \in \Sigma \mid-0.05<x_{3}<0.05$} & \multirow{3}{*}{0.05} & $10^{4}$ & 13 & 21.4641 & 0.0768 \\
\hline & & $10^{6}$ & 14 & 23.6017 & 0.0705 \\
\hline & & $10^{8}$ & 14 & 23.6288 & 0.0704 \\
\hline
\end{tabular}

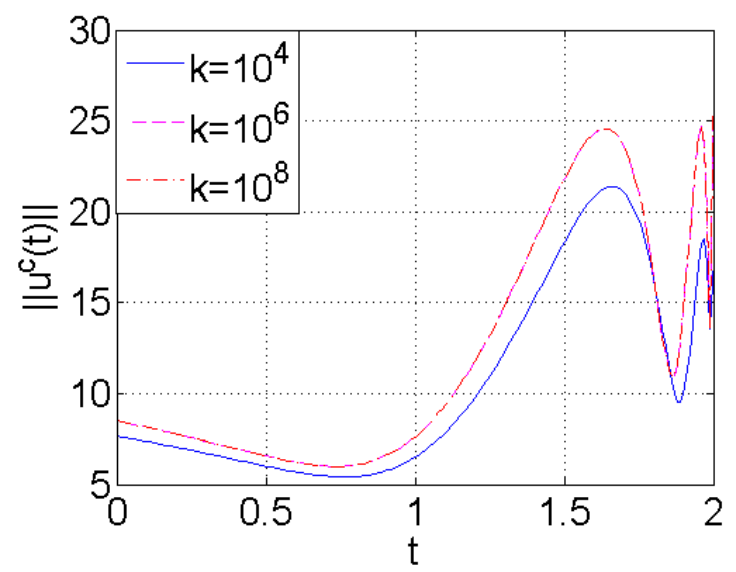

Figure 3. Norm of the computed control vs. time for $k=10^{4}, 10^{6}, 10^{8}$ and $\omega=$ $\left\{\left(x_{1}, x_{2}, x_{3}\right) \in \Sigma \mid x_{3}>0\right\}$

comparing the results in Tables 1 and 2 with those to be shown in Section 6.2 strongly suggests that the exact controllability property holds for $y_{T}$ defined by (6.1) (or, at least, that $y_{T}$ is very close to a function for which the exact controllability property holds).

Figure 3 shows the behavior of the $\mathcal{L}^{2}$-norm of $u^{c}(t)$ versus $t$ for several values of $k$, assuming that $\left\{\left(x_{1}, x_{2}, x_{3}\right) \in \Sigma \mid x_{3}>0\right\}$; the oscillations of the control function as $t$ is nearing $T$ (a well-known phenomenon for the usual heat equation as shown in, e.g., $[10,12,16])$ appear clearly on this figure. On Figure 4, we have visualized $y^{c}(T)$ and $\left|y_{T}-y^{c}(T)\right|$, assuming that $k=10^{8}$ and $\omega=\left\{\left(x_{1}, x_{2}, x_{3}\right) \in \Sigma \mid x_{3}>0\right\}$ : not surprisingly, the matching between $y^{c}(T)$ and $y_{T}$ is better on $\omega$ than on $\Sigma \backslash \omega$.

An important issue that we are going to address now (with $y_{T}$ still defined by (6.1)) is the influence of the diffusion coefficient $\mu$ : Qualitatively speaking, increasing $\mu$ will enhance the 'natural' tendency of the system which is (if left uncontrolled) to have $y(t) \rightarrow \bar{y}_{0}\left(=\int_{\Sigma} y_{0} \mathrm{~d} \Sigma /|\Sigma|\right)$ as $t \rightarrow \infty$, an effect that one has to 'fight' when trying to control the system (unless $y_{T}=\overline{y_{0}}$ ); on the other hand, if $\mu=0$, the absence of diffusion will prevent 

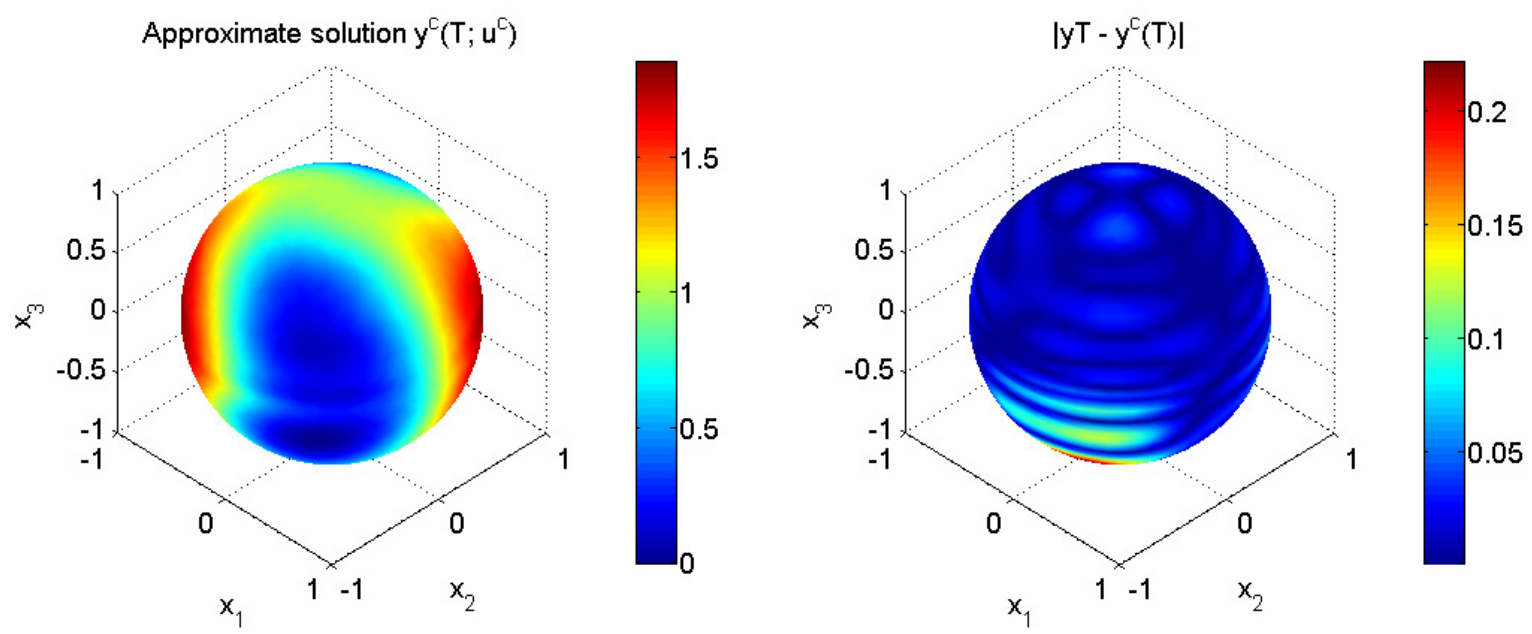

Figure 4. Visualization of $y^{c}(T)$ (left) and $\left|y_{T}-y^{c}(T)\right|($ right $)\left(k=10^{8}\right.$ and $\omega=\left\{\left(x_{1}, x_{2}, x_{3}\right) \in\right.$ $\left.\left.\Sigma \mid x_{3}>0\right\}\right)$.

TABLE 3. Influence of $\mu$.

\begin{tabular}{cccc}
\hline$\mu$ & No. Iters & Norm $u^{c}$ & Rel. error \\
\hline 1 & 44 & 24.3525 & 0.0634 \\
$1 / 2$ & 34 & 19.3586 & 0.0566 \\
$1 / 4$ & 46 & 17.8515 & 0.0635 \\
$1 / 8$ & 100 & 36.8160 & 0.0833 \\
\hline
\end{tabular}

the control to act in $\Sigma \backslash \omega$. From these obvious considerations we anticipate controllability to be a non-monotone function of $\mu$, everything else being the same. The results reported in Table 3 validate the above prediction; they have been obtained using $y_{0}=0, h=0.0051, \Delta t=1 / 100, k=10^{8}, T=2$, and $\omega=\left\{\left(x_{1}, x_{2}, x_{3}\right) \in \Sigma \mid x_{3}>0\right\}$.

\subsection{Results for non-zero Lipschitz continuous target functions}

For the second series of numerical experiments, we take for $y_{T}$ the Lipschitz continuous target function defined by

$$
y_{T}(x)=\left|\sin \left(2 x_{1} x_{2}\right)\right|+\cos \left(2 x_{2} x_{3}\right), \quad \forall\left(x_{1}, x_{2}, x_{3}\right) \in \Sigma .
$$

We choose again $R=1$ and $y_{0}=0$. Concerning the other parameters we take $T=2$ as final time, $\mathbf{u}_{0}=0$, $k=10^{8}, \mu=1 / 2$, and $t o l=10^{-5}$. The first numerical results we present have been obtained with $\omega=$ $\left\{\left(x_{1}, x_{2}, x_{3}\right) \in \Sigma \mid-0.3<x_{3}<0.3\right\}$ and several values of $h$ and $\Delta t$; these results have been reported in Table 4 . As with $y_{T}$ defined by (6.1), these results show convergence when $h$ and $\Delta t \longrightarrow 0$.

Next, we are going to investigate the influence of $\omega$ on the controllability. For the related experiments, we took $h=0.0051$ (4098 grid points) and $\Delta t=1 / 100$, the other parameter and data staying as above. The results of the corresponding numerical experiments have been reported in Table 5 . We observe with interest that although the target function defined by (6.2) is less smooth than the one defined by (6.1), it has comparable controllability properties.

We have visualized on Figure 5, for $\omega=\left\{\left(x_{1}, x_{2}, x_{3}\right) \in \Sigma \mid-0.3<x_{3}<0.3\right\}$, the computed final solution $y^{c}(T)$ (left) and the matching error $\left|y_{T}-y^{c}(T)\right|($ right $)$. 
TABLE 4. Summary of convergence results (target function (6.2) and $\omega=\left\{\left(x_{1}, x_{2}, x_{3}\right) \in \Sigma \mid\right.$ $\left.\left.-0.3<x_{3}<0.3\right\}\right)$.

\begin{tabular}{ccccccc}
\hline$h$ & 0.0787 & 0.0201 & 0.0051 & 0.0013 & 0.0201 & 0.0051 \\
$\Delta t$ & $1 / 100$ & $1 / 100$ & $1 / 100$ & $1 / 100$ & $1 / 200$ & $1 / 200$ \\
\hline No. iters & 6 & 12 & 10 & 9 & 12 & 10 \\
Norm $u^{c}$ & 6.6679 & 8.8285 & 9.2332 & 9.6992 & 8.7940 & 9.1684 \\
Rel. error & 0.0671 & 0.0638 & 0.0674 & 0.0678 & 0.0635 & 0.0676 \\
\hline
\end{tabular}

TABLE 5. Numerical results for different sub-domains $\omega$ (target function (6.2)).

\begin{tabular}{ccccc}
\hline$\omega$ & $|\omega| /|\Sigma|$ & No. Iters & Norm $u^{c}$ & Rel. error \\
\hline$\Sigma$ & 1 & 14 & 6.0928 & 0.0022 \\
$\left\{\left(x_{1}, x_{2}, x_{3}\right) \in \Sigma \mid-0.9<x_{3}<0.9\right\}$ & 0.90 & 8 & 5.5072 & 0.0146 \\
$\left\{\left(x_{1}, x_{2}, x_{3}\right) \in \Sigma \mid-0.8<x_{3}<0.8\right\}$ & 0.80 & 8 & 5.5881 & 0.0222 \\
$\left\{\left(x_{1}, x_{2}, x_{3}\right) \in \Sigma \mid-0.7<x_{3}<0.7\right\}$ & 0.70 & 10 & 5.7698 & 0.0341 \\
$\left\{\left(x_{1}, x_{2}, x_{3}\right) \in \Sigma \mid-0.55<x_{3}<0.55\right\}$ & 0.55 & 13 & 7.0761 & 0.0414 \\
$\left\{\left(x_{1}, x_{2}, x_{3}\right) \in \Sigma \mid-0.5<x_{3}<0.5\right\}$ & 0.50 & 9 & 7.1176 & 0.0497 \\
$\left\{\left(x_{1}, x_{2}, x_{3}\right) \in \Sigma \mid-0.3<x_{3}<0.3\right\}$ & 0.30 & 10 & 9.2332 & 0.0674 \\
$\left\{\left(x_{1}, x_{2}, x_{3}\right) \in \Sigma \mid-0.2<x_{3}<0.2\right\}$ & 0.20 & 12 & 12.6560 & 0.0645 \\
$\left\{\left(x_{1}, x_{2}, x_{3}\right) \in \Sigma \mid-0.05<x_{3}<0.05\right\}$ & 0.05 & 13 & 22.4331 & 0.0679 \\
\hline
\end{tabular}
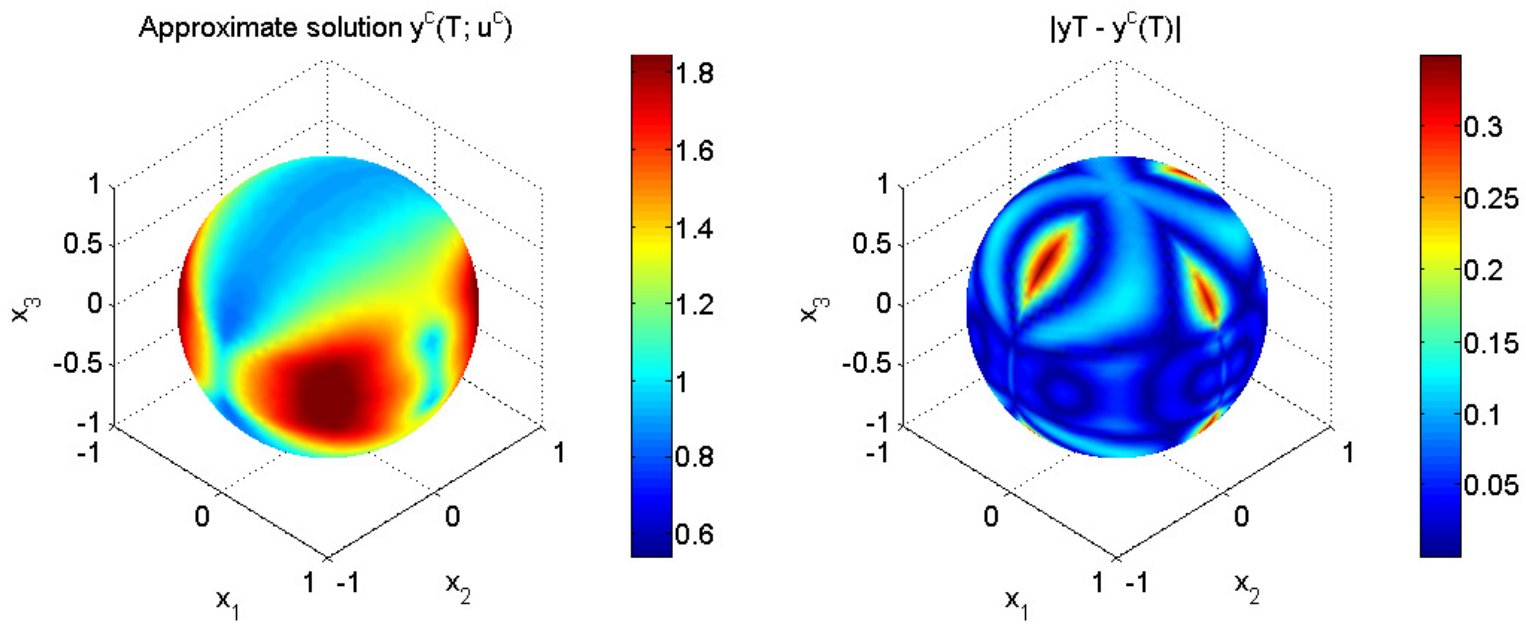

Figure 5. Case $\omega=\left\{\left(x_{1}, x_{2}, x_{3}\right) \in \Sigma \mid-0.3<x_{3}<0.3\right\}$ Visualization of $y^{c}(T)$ (left) and $\left|y_{T}-y^{c}(T)\right|($ right $)$.

The next investigation, with the target function defined by (6.2), concerns the influence of $\mu$, for various control domains $\omega$, the other parameters and data being the same as above.

The results reported in Table 6 show that if $\mu$ increases beyond some critical value, controllability deteriorates, a property we have been expecting. Similarly, controllability deteriorates if $\mu$ decreases below some critical value.

The influence of $T$ on the controllability has been investigated, using different values for $\mu, h=0.0051$, $\Delta t=1 / 100, k=10^{8}$, and $\omega=\left\{\left(x_{1}, x_{2}, x_{3}\right) \in \Sigma \mid-0.3<x_{3}<0.3\right\}$, the other data being like in the previous experiment. The results presented in Table 7 , show that, as expected, increasing $T$ enhances controllability. 
TABLE 6. Influence of $\mu$ on the controllability (target function (6.2)).

\begin{tabular}{cccccc}
\hline$\omega$ & $|\omega| /|\Sigma|$ & $\mu$ & No. Iters & Norm $u^{c}$ & Rel. error \\
\hline & & 1 & 20 & 12.5932 & 0.0779 \\
$\left\{\left(x_{1}, x_{2}, x_{3}\right) \in \Sigma \mid x_{3}>0\right\}$ & & $1 / 2$ & 22 & 11.7713 & 0.0682 \\
& 0.5 & $1 / 4$ & 26 & 12.2879 & 0.0723 \\
& & $1 / 8$ & 55 & 24.4898 & 0.0865 \\
$\left\{\left(x_{1}, x_{2}, x_{3}\right) \in \Sigma \mid-0.5<x_{3}<0.5\right\}$ & $1 / 10$ & 99 & 45.1834 & 0.0883 \\
\hline & & 1 & 13 & 9.7446 & 0.0468 \\
& & $1 / 2$ & 9 & 7.1176 & 0.0497 \\
& & $1 / 4$ & 11 & 6.4009 & 0.0511 \\
$\left\{\left(x_{1}, x_{2}, x_{3}\right) \in \Sigma \mid-0.3<x_{3}<0.3\right\}$ & & $1 / 10$ & 20 & 9.0149 & 0.0537 \\
& 0.3 & $1 / 4$ & 11 & 11.9749 & 0.0684 \\
& & $1 / 8$ & 11 & 8.7718 & 0.0690 \\
& & $1 / 10$ & 30 & 45.1834 & 0.0832 \\
\hline
\end{tabular}

TABLE 7. Influence of $T$ and $\mu$ on the controllability $\left(\omega=\left\{\left(x_{1}, x_{2}, x_{3}\right) \in \Sigma \mid-0 .<x_{3}<0.3\right\}\right)$.

\begin{tabular}{|c|c|c|c|c|c|}
\hline$\mu$ & $T$ & $\overline{|\omega| /|\Sigma|}$ & No. Iters & Norm $u^{c}$ & Rel. error \\
\hline \multirow{6}{*}{1} & 2 & 0.3 & 11 & 11.9749 & 0.0684 \\
\hline & 1.5 & 0.3 & 10 & 12.2146 & 0.0688 \\
\hline & 1 & 0.3 & 10 & 13.3376 & 0.0676 \\
\hline & $1 / 2$ & 0.3 & 11 & 17.7431 & 0.0699 \\
\hline & $1 / 4$ & 0.3 & 18 & 43.5057 & 0.0843 \\
\hline & $1 / 8$ & 0.3 & 65 & 143.4465 & 0.1696 \\
\hline \multirow{6}{*}{$1 / 2$} & 4 & 0.3 & 7 & 7.0106 & 0.0793 \\
\hline & 3 & 0.3 & 7 & 7.2707 & 0.0790 \\
\hline & 2 & 0.3 & 10 & 9.2332 & 0.0674 \\
\hline & 1 & 0.3 & 11 & 12.3766 & 0.0693 \\
\hline & $1 / 2$ & 0.3 & 16 & 29.0787 & 0.0843 \\
\hline & $1 / 4$ & 0.3 & 50 & 116.5275 & 0.1352 \\
\hline \multirow{6}{*}{$1 / 4$} & 8 & 0.3 & 7 & 4.9572 & 0.0793 \\
\hline & 6 & 0.3 & 7 & 5.1332 & 0.0791 \\
\hline & 4 & 0.3 & 10 & 6.4831 & 0.0674 \\
\hline & 2 & 0.3 & 11 & 8.7718 & 0.0690 \\
\hline & 1 & 0.3 & 24 & 22.1714 & 0.0784 \\
\hline & $1 / 2$ & 0.3 & 51 & 73.9656 & 0.1398 \\
\hline \multirow{6}{*}{$1 / 8$} & 16 & 0.3 & 7 & 3.4923 & 0.0795 \\
\hline & 12 & 0.3 & 7 & 3.6297 & 0.0791 \\
\hline & 8 & 0.3 & 10 & 4.5842 & 0.0674 \\
\hline & 4 & 0.3 & 11 & 8.7718 & 0.0690 \\
\hline & 2 & 0.3 & 20 & 24.4898 & 0.0812 \\
\hline & 1 & 0.3 & 50 & 49.6053 & 0.1431 \\
\hline
\end{tabular}


TABLE 8. Numerical result for $T=1 / 5, \mu=1 / 2$, and $\omega=\left\{\left(x_{1}, x_{2}, x_{3}\right) \in \Sigma \mid-0.65<x_{3}<0.65\right\}$.

\begin{tabular}{cccccc}
\hline$\mu$ & $T$ & $|\omega| /|\Omega|$ & No. Iters & Norm $u^{c}$ & Rel. error \\
\hline $1 / 2$ & $1 / 5$ & 0.65 & 32 & 36.0589 & 0.0688 \\
\hline
\end{tabular}
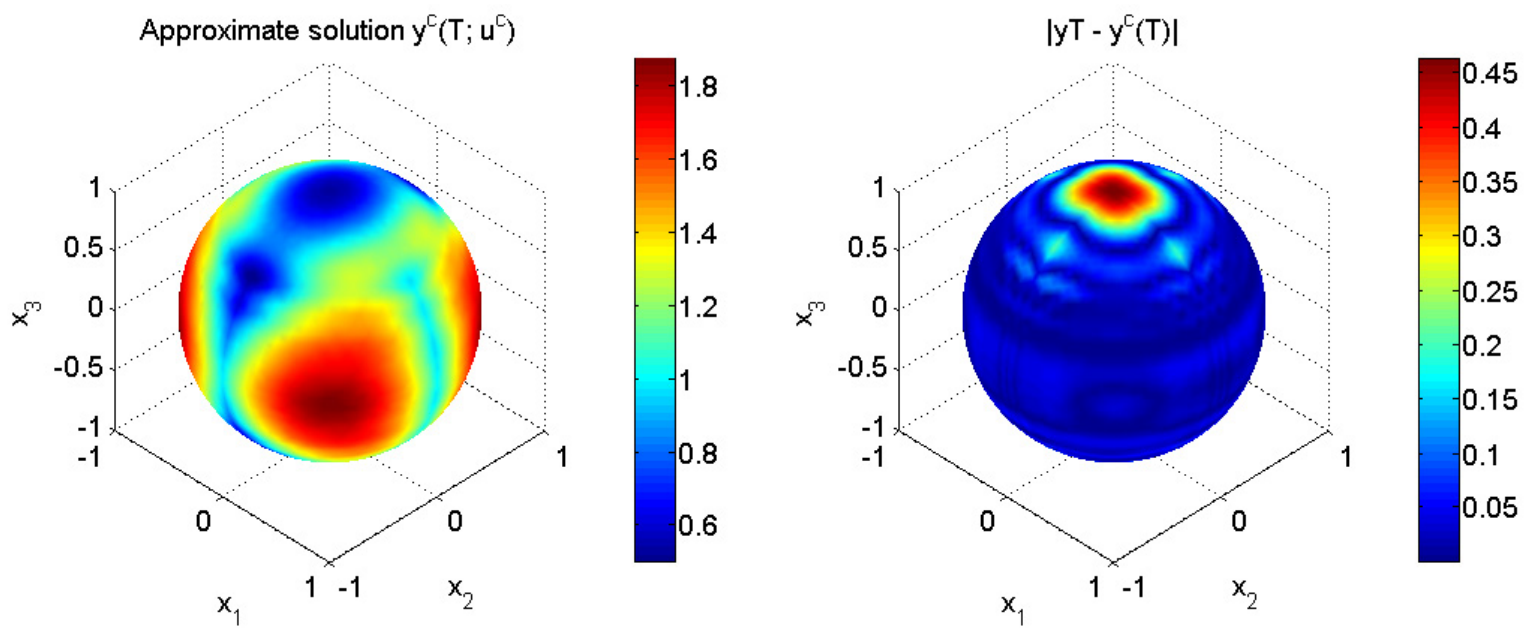

Figure 6. Case: $\mu=1 / 2, T=1 / 5$, and $\omega=\left\{\left(x_{1}, x_{2}, x_{3}\right) \in \Sigma \mid-0.65<x_{3}<0.65\right\}$. Visualization of $y^{c}(T)$ (left) and $\left|y_{T}-y^{c}(T)\right|$ (right).

TABle 9. Numerical results for different control domains of equal area.

\begin{tabular}{ccccc}
\hline Supporting set $\omega$ & $|\omega| /|\Omega|$ & No. Iters & Norm $u^{c}$ & Rel. error \\
\hline$\omega_{1}=\left\{\left(x_{1}, x_{2}, x_{3}\right) \in \Sigma \mid x_{3}>0\right\}$ & 0.5 & 22 & 11.7713 & 0.0682 \\
$\omega_{2}=\left\{\left(x_{1}, x_{2}, x_{3}\right) \in \Sigma \mid-0.5<x_{3}<0.5\right\}$ & 0.5 & 9 & 7.1176 & 0.0497 \\
$\omega_{3}=\left\{\left(x_{1}, x_{2}, x_{3}\right) \in \Sigma \mid-0.5<x_{1}<0.5\right\}$ & 0.5 & 14 & 9.9366 & 0.0598 \\
\hline$\omega_{4}=\left\{\left(x_{1}, x_{2}, x_{3}\right) \in \Sigma \mid 0.6<x_{3}<1\right\}$ & 0.2 & 24 & 17.2843 & 0.1358 \\
$\omega_{5}=\left\{\left(x_{1}, x_{2}, x_{3}\right) \in \Sigma \mid-0.2<x_{3}<0.2\right\}$ & 0.2 & 12 & 12.6560 & 0.0645 \\
$\omega_{6}=\left\{\left(x_{1}, x_{2}, x_{3}\right) \in \Sigma \mid-0.2<x_{1}<0.2\right\}$ & 0.2 & 20 & 16.8209 & 0.0613 \\
\hline
\end{tabular}

The parameters $T$ and $\mu$ being given, a systematic way to enhance controllability is to increase $|\omega|$ as shown by Table 8, which corresponds to $\mu=1 / 2, T=1 / 5$ and $\omega=\left\{\left(x_{1}, x_{2}, x_{3}\right) \in \Sigma \mid-0.65<x_{3}<0.65\right\}$. On Figure 6 (left), we have visualized $y^{c}(T)$, while the controllability gap $\left|y_{T}-y^{c}(T)\right|$ has been visualized on Figure 6 (right). Not surprisingly the control deteriorates away from $\omega$, the controllability gap taking its maximal values at the poles.

To conclude with the target function (6.2), we are going to compare the controllability properties associated with control domains $\omega$ of same area but with different shapes or location. For these experiments we take $\mu=1 / 2$ and $T=2$, the other parameters being as in the above experiments. Table 9 summarizes the results obtained from two sets of experiments: In the first case (resp., the second case) we have three control domains $\omega$ with $|\omega|=2 \pi$ (resp., $|\omega|=0.8 \pi$ ). These results show that the number of conjugate gradient iterations necessary to achieve convergence may vary significantly, as does the controllability gap for the domains of smaller area. On the other hand, the norm of the computed optimal control shows less variations. 
TABLE 10. Numerical results with the Lipschitz continuous target function (6.3) for various values of $\kappa_{N}$.

\begin{tabular}{ccccc}
\hline$\kappa_{N}$ & $\left|\omega_{N}\right| /|\Sigma|$ & No. Iters & Norm $u^{c}$ & Rel. error \\
\hline 0 & 0.5 & 141 & 111.8703 & 0.1559 \\
0.75 & 0.125 & 300 & 142.3226 & 0.2384 \\
0.875 & 0.0625 & 250 & 243.3815 & 0.2053 \\
0.95 & 0.025 & 315 & 327.3470 & 0.2842 \\
\hline
\end{tabular}

TABLE 11. Numerical results with the Lipschitz continuous target function (6.3) for $\omega=\omega_{N} \cup \omega_{S}$ and various values of $\kappa_{S}=\kappa_{N}$.

\begin{tabular}{ccccc}
\hline$\kappa_{N}=\kappa_{S}$ & $|\omega| /|\Sigma|$ & No. Iters & Norm $u^{c}$ & Rel. error \\
\hline 0.5 & 0.5 & 82 & 72.3659 & 0.1064 \\
0.75 & 0.25 & 106 & 127.5446 & 0.1529 \\
0.875 & 0.125 & 149 & 246.4855 & 0.1624 \\
0.950 & 0.050 & 127 & 284.5191 & 0.1886 \\
0.975 & 0.025 & 165 & 571.7372 & 0.1808 \\
\hline
\end{tabular}

The last experiments with a Lipschitz continuous target function concern $y_{T}$ defined by

$$
y_{T}(x)=\left\{\begin{array}{cl}
2 & \text { if } x \in \Sigma, x_{3}>\frac{1}{6}, \\
\frac{3}{2} \sin \left(3 \pi x_{3}\right)+\cos \left(2 \pi x_{3}\right) & \text { if } x \in \Sigma,-\frac{1}{6} \leq x_{3} \leq \frac{1}{6}, \\
-1 & \text { if } x \in \Sigma, x_{3}<-\frac{1}{6} .
\end{array}\right.
$$

For these experiments we took $R=1, y_{0}=0, T=2, \mu=1 / 2, \mathbf{u}_{0}=0, k=10^{10}, \Delta t=1 / 100, h=0.0051$, and tol $=10^{-5}$ for the stopping criterion. We considered first control domains $\omega=\omega_{N}$ of the following type:

$$
\omega_{N}=\left\{\left(x_{1}, x_{2}, x_{3}\right) \in \Sigma, \quad \kappa_{N}<x_{3} \leq 1\right\}
$$

with $0 \leq \kappa_{N}<1$. The results of numerical experiments with $\kappa_{N}$ varying from 0.5 to 0.95 are reported in Table 10. They show that getting close to $y_{T}$ is more energy-demanding than with the target function defined by (6.2); also, for the target function (6.3) at least, using cap shaped control domains $\omega$ makes controllability more difficult, for the same value of $|\omega|$. To test this last property we modified $\omega$, defining it now as $\omega=\omega_{N} \cup \omega_{S}$, with

$$
\omega_{S}=\left\{\left(x_{1}, x_{2}, x_{3}\right) \in \Sigma, \quad-1 \leq x_{3}<-\kappa_{S}\right\},
$$

and $0 \leq \kappa_{S}<1$. On Figure 7 (left) (resp., (right)) we have visualized the function $y^{c}(T)$ (resp., the controllability gap $\left.\left|y_{T}-y^{c}(T)\right|\right)$ associated with the computed optimal control, assuming that $\omega=\omega_{N}$, with $\kappa_{N}=0$.

On Table 11, we have reported the numerical results associated with $\omega=\omega_{N} \cup \omega_{S}, \kappa_{N}=\kappa_{S}, \kappa_{N}$ varying from 0.5 to 0.975 (that is, $|\omega| /|\Sigma|$ varying from 0.5 to 0.025 ).

By comparison with Table 10, these results show that for the same area the two cap control sets have better controllability properties than the one cap ones, which is not surprising since by splitting $\omega$ the way we did we have reduced the mean distance to $\omega$ of the points of $\Sigma \backslash \omega$ (actually, results in that direction have been obtained in [18] for domains of $\mathbb{R}^{d}$ with the flat metric, suggesting that the controllability enhancement by fragmentation of $\omega$, we observe here, is not specific to the sphere). 

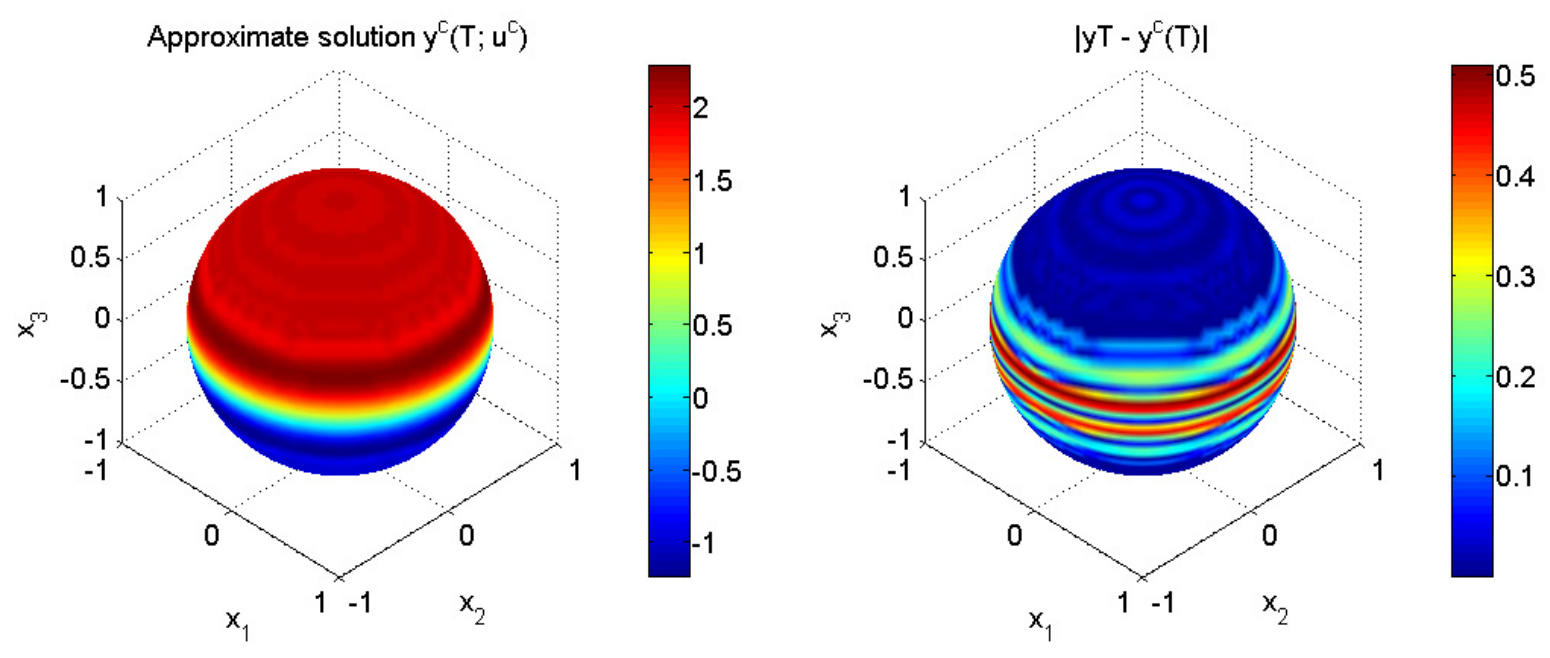

Figure 7. Target function (6.3): Case $\omega=\omega_{N}$ with $\kappa_{N}=0$. Visualization of $y^{c}(T)$ (left) and $\left|y_{T}-y^{c}(T)\right|($ right $)$.

TABLE 12. Numerical results with the discontinuous target function $(6.6)(h=0.0051, \Delta t=1 / 100)$.

\begin{tabular}{cccccc}
\hline$\kappa_{N}$ & $\left|\omega_{N}\right| /|\Sigma|$ & $\mathrm{k}$ & No. Iters & Norm $u^{c}$ & Rel. error \\
\hline \multirow{4}{*}{0} & & $10^{6}$ & 72 & 148.8302 & 0.1402 \\
& \multirow{2}{*}{0.5} & $10^{8}$ & 80 & 117.8068 & 0.1274 \\
& & $10^{10}$ & 81 & 172.6996 & 0.1270 \\
0.75 & $10^{15}$ & 81 & 172.7599 & 0.1271 \\
\hline \multirow{3}{*}{0.125} & $10^{8}$ & 140 & 99.2490 & 0.3193 \\
& & $10^{10}$ & 150 & 101.7040 & 0.3180 \\
& & $10^{15}$ & 150 & 101.8320 & 0.3180 \\
\hline
\end{tabular}

\subsection{Results for a discontinuous target function}

The target functions defined by (6.1), (6.2) and (6.3) are all Lipschitz continuous. The test problem that we are going to consider now is more challenging since this time the target function $y_{T}$ is the discontinuous function defined by

$$
y_{T}(x)=\left\{\begin{aligned}
2 \text { if } x \in \Sigma, & x_{3}>0 \\
-1 \text { if } x \in \Sigma, & x_{3}<0
\end{aligned}\right.
$$

As for the above cases, $\Sigma$ is the sphere of radius one centered at $(0,0,0)$, the other data being, in addition to $y_{T}$, $y_{0}=0, T=2, \mu=1 / 2, \mathbf{u}_{0}=0, \Delta t=1 / 100, h=0.0051, t o l=10^{-5}, \omega=\omega_{N}$ with $\omega_{N}$ as in (6.4). Table 12 shows numerical results obtained for two supporting sub-domains $\omega_{N}$ and various values of $k$. We observe that for $k>10^{10}$ the results are essentially identical; on the other hand, the size of $\omega$ has a very significant influence on the number of iterations, the cost of the control, and how close $y(T)$ is to the target function. Concerning this last issue it is clear that the discontinuity of the target function in (6.6) makes controllability much more difficult.

For $\kappa_{N}=0$, we have visualized on Figure 8 the computed solution $y^{c}(T)$ (left) and the controllability gap $\left|y^{c}(T)-y T\right|$ (right). This figure shows that away from the target function discontinuity set (the equatorial circle $x_{3}=0$, here) the matching between $y^{c}(T)$ and $y_{T}$ is quite 'reasonable'. In fact, the relative error is mostly 

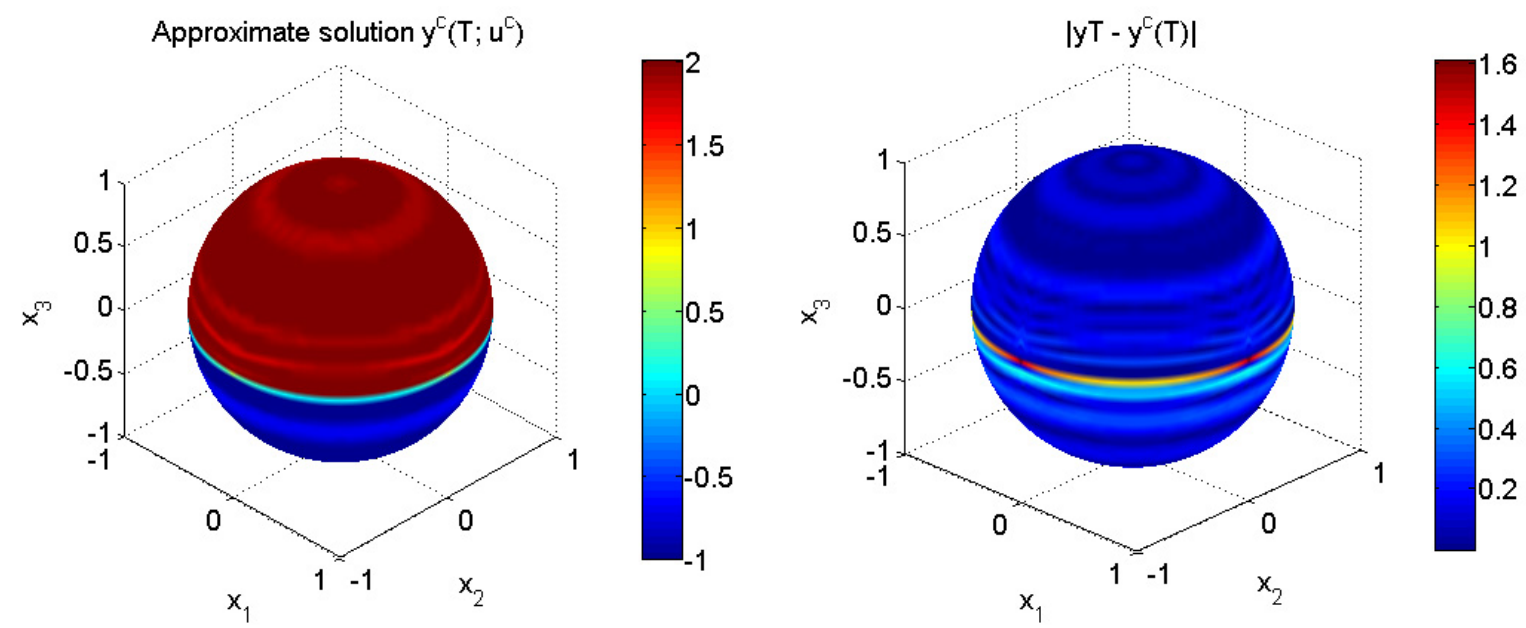

Figure 8. Target function (6.6): Case $\omega=\omega_{N}$ with $\kappa_{N}=0$. Visualization of $y^{c}(T)$ (left) and $\left|y_{T}-y^{c}(T)\right|($ right $)$.

TABLE 13. Numerical results for different values of $k$ with a discontinue target function, supporting $\omega_{N} ; \Delta t=1 / 200$ and $h=0.0017$.

\begin{tabular}{cccccc}
\hline$\kappa_{N}$ & $\left|\omega_{N}\right| /|\Sigma|$ & $\mathrm{k}$ & No. Iters & Norm $u^{c}$ & Rel. error \\
\hline \multirow{4}{*}{0} & & $10^{6}$ & 90 & 195.8025 & 0.1451 \\
& 0.5 & $10^{8}$ & 90 & 198.9890 & 0.1379 \\
& & $10^{10}$ & 90 & 199.0279 & 0.1378 \\
& $10^{15}$ & 90 & 199.0131 & 0.1378 \\
\hline \multirow{3}{*}{0.75} & \multirow{2}{*}{0.125} & $10^{6}$ & 150 & 187.1418 & 0.2927 \\
& & $10^{8}$ & 200 & 207.7558 & 0.2881 \\
& & $10^{10}$ & 216 & 218.4220 & 0.2880 \\
& & $10^{15}$ & 216 & 218.4223 & 0.2880 \\
\hline
\end{tabular}

concentrated near the discontinuity, since

$$
\left(\int_{\Sigma_{0.8}}\left|y^{c}(T)-y_{T}\right|^{2} \mathrm{~d} \Sigma / \int_{\Sigma_{0.8}}\left|y_{T}\right|^{2} \mathrm{~d} \Sigma\right)^{1 / 2}=0.03, \quad \text { where } \quad \Sigma_{0.8}=\left\{\left(x_{1}, x_{2}, x_{3}\right) \in \Sigma|| x_{3} \mid>0.2\right\} .
$$

We repeated these experiments with $h=0.0017$ and $\Delta t=1 / 200$ : The results reported in Table 13 are close to those in Table 12.

\subsection{Null controllability problems}

The following numerical experiments concern the target function $y_{T}=0$, that is the null state. The experiments reported in Section 6.4.1 concern smooth initial state functions $y_{0}$, while in Section 6.4.2, we investigatenull controllability when $y_{0}$ is a discontinuous function.

\subsubsection{Null-controllability for smooth function $y_{0}$}

In this section, the first initial state $y_{0}$ that we consider is defined by

$$
y_{0}\left(x_{1}, x_{2}, x_{3}\right)=\sin \left(2 x_{1} x_{2}\right)+\cos \left(2 x_{2} x_{3}\right), \quad \forall\left(x_{1}, x_{2}, x_{3}\right) \in \Sigma,
$$


TABle 14. Numerical results for the initial data (6.7) $\left(\omega=\left\{\left(x_{1}, x_{2}, x_{3}\right) \in \Sigma|| x_{3} \mid<0.3\right\}\right)$.

\begin{tabular}{ccccc}
\hline Quantity & $T=1$ & $T=2$ & $T=3$ & $T=5$ \\
\hline Number of iterations & 33 & 12 & 12 & 10 \\
Norm $u^{c}$ & 11.0765 & 4.9177 & 3.6459 & 2.6355 \\
$\left\|y^{c}(T)\right\| \mathcal{L}_{\mathcal{L}^{2}(\Sigma)}$ & 0.0305 & 0.0247 & 0.0142 & 0.0091 \\
$\left\|y^{c}(T)\right\|_{\mathcal{L}^{2}(\Sigma)} /\left\|y_{0}\right\|_{\mathcal{L}^{2}(\Sigma)}$ & 0.0086 & 0.0070 & 0.0040 & 0.0026 \\
$\left\|y^{c}(T)\right\|_{\mathcal{L}^{2}(\Sigma)} /\left\|y_{n c}^{c}(T)\right\|_{\mathcal{L}^{2}(\Sigma)}$ & 0.0098 & 0.0080 & 0.0046 & 0.0029 \\
\hline
\end{tabular}

TABLE 15. Numerical result for the initial data (6.7): dependence of $\omega$.

\begin{tabular}{ccccccc}
\hline$\omega$ & $|\omega| /|\Sigma|$ & $N$. Iter & Norm $u^{c}$ & $\left\|y^{c}(T)\right\|_{\mathcal{L}^{2}(\Sigma)}$ & $\frac{\left\|y^{c}(T)\right\|_{\mathcal{L}^{2}(\Sigma)}}{\left\|y_{0}\right\|_{\mathcal{L}^{2}(\Sigma)}}$ & $\frac{\left\|y^{c}(T)\right\|_{\mathcal{L}^{2}(\Sigma)}}{\left\|y_{n c}^{c}(T)\right\|_{\mathcal{L}^{2}(\Sigma)}}$ \\
\hline$\omega_{1}$ & 1 & 2 & 2.1928 & $1.6726 \times 10^{-6}$ & $4.7226 \times 10^{-7}$ & $5.4073 \times 10^{-7}$ \\
$\omega_{2}$ & 0.90 & 10 & 2.3515 & 0.0110 & 0.0031 & 0.0036 \\
$\omega_{3}$ & 0.70 & 11 & 2.9446 & 0.0191 & 0.0054 & 0.0062 \\
$\omega_{4}$ & 0.55 & 14 & 3.4724 & 0.0232 & 0.0065 & 0.0075 \\
$\omega_{5}$ & 0.30 & 12 & 4.9177 & 0.0247 & 0.0070 & 0.0080 \\
$\omega_{6}$ & 0.20 & 13 & 5.7826 & 0.0284 & 0.0080 & 0.0092 \\
\hline
\end{tabular}

the set $\Sigma$ still being the sphere of radius one centered at $(0,0,0)$. We observe that the mean value of $y_{0}$ is strictly positive and given by $\bar{y}_{0}=\int_{\Sigma} \cos \left(2 x_{2} x_{3}\right) \mathrm{d} \Sigma /(4 \pi)$, implying that if the system is left uncontrolled, the function $y(t)$ will converge to the positive constant $\bar{y}_{0}$ as $t \longrightarrow+\infty$.

The numerical experiments reported below have been performed using $\Delta t=1 / 100, h=0.0051, k=10^{8}$. The conjugate gradient algorithm $(5.25)-(5.37)$ has been initialized with $\mathbf{u}_{0}=\mathbf{0}$ and stopped using tol $=$ $5 \times 10^{-7}$ in the stopping criterion. The results reported in Table 14 correspond to $\mu=1 / 2, T=1,2,3,5$ and $\omega=\left\{\left(x_{1}, x_{2}, x_{3}\right) \in \Sigma|| x_{3} \mid<0.3\right\}$. In the first column of Table 14, we have denoted by $y_{n c}^{c}(T)$ the computed value of the state function associated with $\mathbf{v}=\mathbf{0}$ (the uncontrolled situation).

As expected, the norms of the control and of the computed value of $y(T)$ are decreasing functions of $T$. The last two rows of Table 14 show that indeed approximate null-controllability is taking place, the last row being particularly interesting since it shows that the control process has 'almost killed' (that is considerably damped) the constant component of the solution. In order to study the influence of $\omega$ on the controllability, we ran a series of experiments for $T=2$ and various control sets $\omega$, the other data and parameters being unchanged. The sets $\omega$ we considered are given by:

$$
\begin{aligned}
& \omega_{1}=\Sigma \\
& \omega_{2}=\left\{\left(x_{1}, x_{2}, x_{3}\right) \in \Sigma|| x_{3} \mid<0.9\right\} \\
& \omega_{3}=\left\{\left(x_{1}, x_{2}, x_{3}\right) \in \Sigma|| x_{3} \mid<0.7\right\} \\
& \omega_{4}=\left\{\left(x_{1}, x_{2}, x_{3}\right) \in \Sigma|| x_{3} \mid<0.55\right\} \\
& \omega_{5}=\left\{\left(x_{1}, x_{2}, x_{3}\right) \in \Sigma|| x_{3} \mid<0.3\right\} \\
& \left.\omega_{6}=\left\{\left(x_{1}, x_{2}, x_{3}\right) \in \Sigma|| x_{3} \mid<0.2\right\}\right)
\end{aligned}
$$

the corresponding results being reported in Table 15. They show, as already observed, that increasing $|\omega|$ enhances controllability; they show also very spectacular controllability results if $\omega=\Sigma$, a not very realistic situation indeed.

On Figure 9 we have visualized, for $\omega=\omega_{5}$, the time evolution of the $\mathcal{L}^{2}(\omega)$-norm of the computed optimal control (left figure), and (on the right figure) the point-wise values at $t=T$ of the associated state function 

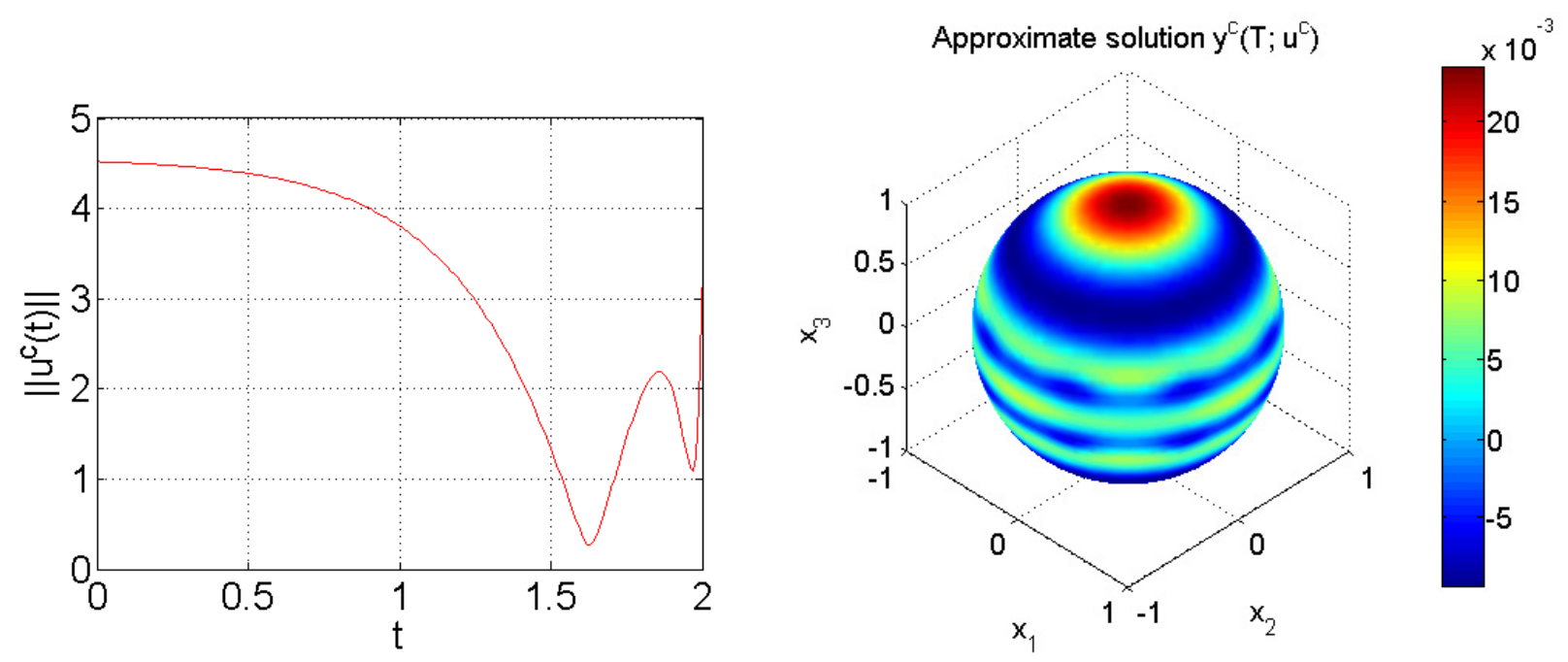

Figure 9. Left: Time evolution of the $\mathcal{L}^{2}(\omega)$-norm of the optimal control. Right: Visualization of the point-wise values of the final state function $y^{c}\left(T ; u^{c}\right)$ (initial data $(6.7), \omega=\omega_{5}, T=2$ ).

TABLE 16. Numerical results for the initial data (6.8) $\left(\omega=\left\{\left(x_{1}, x_{2}, x_{3}\right) \in \Sigma|| x_{3} \mid<0.3\right\}\right)$.

\begin{tabular}{ccccc}
\hline Quantity & $T=1$ & $T=2$ & $T=3$ & $T=5$ \\
\hline Number of iterations & 23 & 26 & 37 & 40 \\
Norm $u^{c}$ & 0.5623 & 0.0288 & 0.0015 & $4.0466 \times 10^{-6}$ \\
$\left\|y^{c}(T)\right\| \|_{\mathcal{L}^{2}(\Sigma)}$ & 0.0024 & $1.2170 \times 10^{-4}$ & $5.4987 \times 10^{-6}$ & $1.4528 \times 10^{-8}$ \\
$\left\|y^{c}(T)\right\|_{\mathcal{L}^{2}(\Sigma)} /\left\|y_{0}\right\|_{\mathcal{L}^{2}(\Sigma)}$ & 0.0014 & $7.3321 \times 10^{-5}$ & $3.3429 \times 10^{-6}$ & $8.7526 \times 10^{-9}$ \\
$\left\|y^{c}(T)\right\|_{\mathcal{L}^{2}(\Sigma)} /\left\|y_{n c}^{c}(T)\right\|_{\mathcal{L}^{2}(\Sigma)}$ & 0.0280 & 0.0277 & 0.0242 & 0.0240 \\
\hline
\end{tabular}

(denoted by $y^{c}\left(T ; u^{c}\right)$ here). One more time, one can observe: (i) the oscillatory behavior of the control as $t$ is getting close to $T$, and (ii) that the proximity of $y^{c}\left(T ; u^{c}\right)$ to 0 deteriorates as the distance to the control set $\omega$ increases. Similar behaviors were observed for the torus, as reported in [12].

Since the controllability results reported in Tables 14 and 15 reflect the fact that the mean value of the function $y_{0}$ defined by (6.7) is different from 0 , it is natural to consider a function $y_{0}$ whose mean value is 0 in order to see if, as expected, the property $\int_{\Sigma} y_{0} \mathrm{~d} \Sigma=0$ enhances null-controllability. The results reported in Table 16, obtained with $\omega=\omega_{5}, t o l=5 \times 10^{-6}$, and $y_{0}$ defined by

$$
y_{0}\left(x_{1}, x_{2} x_{3}\right)=\sin \left(2 x_{1} x_{2}\right), \quad \forall\left(x_{1}, x_{2}, x_{3}\right) \in \Sigma,
$$

confirm this prediction.

\subsubsection{Null-controllability for a discontinuous function $y_{0}$}

In order to further investigate the influence of the initial state $y_{0}$ on the null-controllability, we performed numerical experiments with the discontinuous initial state function $y_{0}$ defined by

$$
y_{T}(x)=\left\{\begin{array}{l}
1 \text { if } x \in \Sigma, x_{3}>0 \\
0 \text { if } x \in \Sigma, x_{3}<0
\end{array}\right.
$$


TABLE 17. Numerical results for the discontinuous initial data (6.9) $\left(\omega=\omega_{N} \cup \omega_{S}\right.$ and $\kappa_{N}=$ $\left.\kappa_{S}=0.75\right)$.

\begin{tabular}{cccc}
\hline Quantity & $T=2$ & $T=3$ & $T=5$ \\
\hline Number of iterations & 16 & 13 & 10 \\
Norm $u^{c}$ & 3.1317 & 2.3123 & 1.6801 \\
$\left\|y^{c}(T)\right\| \|_{\mathcal{L}^{2}(\Sigma)}$ & 0.0124 & 0.0105 & 0.0080 \\
$\left\|y^{c}(T)\right\|_{\mathcal{L}^{2}(\Sigma)} /\left\|y_{0}\right\|_{\mathcal{L}^{2}(\Sigma)}$ & 0.0049 & 0.0042 & 0.0032 \\
$\left\|y^{c}(T)\right\|_{\mathcal{L}^{2}(\Sigma)} /\left\|y_{n c}^{c}(T)\right\|_{\mathcal{L}^{2}(\Sigma)}$ & 0.0068 & 0.0058 & 0.0044 \\
\hline
\end{tabular}

$\Sigma$ still being the sphere of radius one centered at $(0,0,0)$. The numerical experiments reported below have been performed using $\Delta t=1 / 100, h=0.0051, k=10^{10}$. The conjugate gradient algorithm (5.25)-(5.37) has been initialized with $\mathbf{u}_{0}=\mathbf{0}$ and stopped using tol $=5 \times 10^{-7}$ in the stopping criterion. The results reported in Table 17 correspond to $\mu=1 / 2, T=2,3,5$ and $\omega=\omega_{N} \cup \omega_{S}$ with $\kappa_{N}=\kappa_{S}=0.75$ (see (6.4) and (6.5) for the definition of $\omega_{N}$ and $\left.\omega_{S}\right)$. As expected, controllability improves as $T$ increases. We observe also that the discontinuity of $y_{0}$ does not prevent good null-controllability properties: this is not surprising since the highly oscillatory Fourier components of $y_{0}$ associated with the discontinuity are the ones which are the most strongly damped by the diffusion operator.

Acknowledgements. The authors want to acknowledge the financial support from CONACYT (National Council of Science and Technology in Mexico) through a scholarship for the first author, who did a one-year internship at the University of Houston under the supervision of the second author, and also for the support of the network research group Red de Matemáticas y Desarrollo. We acknowledge also the support from the Math Graduate program at Universidad Autónoma Metropolitana, from the Institute for Advanced Studies at the Hong Kong University of Sciences and Technology, and from the Department of Mathematics at the Hong-Kong Baptist University. Finally, the authors would like to thank the two anonymous reviewers for their very careful reading of the manuscript, and for their most valuable comments and suggestions.

\section{REFERENCES}

[1] A. Bonito and R. Glowinski, On the nodal set of the eigenfunctions of the Laplace-Beltrami operator for bounded surfaces in $\mathbb{R}^{3}$; A computational approach. Commun. Pure Appl. Anal. 13 (2014) 2115-2126.

[2] A. Bonito and J. Pasciak, Convergence analysis of variational and non-variational multigrid algorithms for the Laplace-Beltrami operator. Math. Comput. 81 (2012) 1263-1288.

[3] C. Carthel, R. Glowinski and J.L. Lions, On exact and approximate boundary controllabilities for the heat equation: A numerical approach. J. Optim. Theory Appl. 82 (1994) 429-484.

[4] J.M. Coron and A. Fursikov, Global exact controllability of the 2D Navier-Stokes equations on a manifold without boundary. Russian J. Math. Phys. 4 (1996) 429-448.

[5] E. Fernández-Cara and A. Münch, Strong convergent approximations of null controls for the 1D heat equation. SEMA Journal 61 (2013) 49-78.

[6] E. Fernández-Cara and A. Münch, Numerical null controllability of the 1D heat equation: Carleman weights and duality. J. Optim. Theory Appl. 163 (2014) 253-285.

[7] R. Glowinski, Numerical Methods for Nonlinear Variational Problems, 2nd printing (2008). Springer, New York (1984).

[8] R. Glowinski, Finite element methods for incompressible viscous flow. Vol. 9 of Handbook of Numerical Analysis, edited by P.G. Ciarlet and J.L. Lions. North-Holland, Amsterdam (2003) 3-1176.

[9] R. Glowinski and D.C. Sorensen, Computing the eigenvalues of the Laplace-Beltrami operator on the surface of a torus: A numerical approach. In Partial Differential Equations: Modelling and Numerical Simulation. Springer Netherlands (2008) $225-232$.

[10] R. Glowinski, J.L. Lions and J.W. He, Exact and Approximate Controllability for Distributed Parameter Systems: A Numerical Approach. Cambridge University Press, Cambridge, UK (2008).

[11] G. Lebeau and L. Robbiano, Contrôle exact de l'équation de la chaleur. Commun. Partial Differ. Equ. 20 (1995) 335-356. 
[12] D.A. León, R. Glowinski and L. Héctor Juárez, On the controllability of diffusion processes on the surface of a torus: A computational approach. Pacific J. Optim. 11 (2015) 763-790.

[13] J.L. Lions, Optimal Control of Systems Governed by Partial Differential Equations. Springer-Verlag, New York (1971).

[14] L. Miller, Geometric bounds on the grow rate of null-controllability cost for the heat equation in small time. J. Differ. Equ. 204 (2004) 202-226.

[15] L. Miller, Unique continuation estimates for the Laplacian and the heat equation on non-compact manifolds. Math. Res. Lett. 12 (2005) 37-47.

[16] A. Münch and E. Zuazua, Numerical approximation of null controls for the heat equation: Ill-posedness and remedies. Inverse Problems 26 (2010) 085018.

[17] A. Münch and P. Pedregal, Numerical null controllability of the heat equation through a least squares and variational approach. Eur. J. Appl. Math. 25 (2014) 277-306.

[18] Y. Privat, E. Trélat and E. Zuazua, Optimal shape and location of sensors for parabolic equations with random initial data. Arch. Rational Mech. Anal. 216 (2015) 921-981.

[19] E. Zuazua, Control and numerical approximation of the wave and heat equations. In Vol. 3 of Proc. Internat. Congress Math. Madrid, Spain (2006) 1389-1417. 